\title{
Analytic results for two-loop planar master integrals for Bhabha scattering
}

\author{
Claude Duhr, ${ }^{a}$ Vladimir A. Smirnov ${ }^{b, c}$ and Lorenzo Tancredi ${ }^{d}$ \\ ${ }^{a}$ Bethe Center for Theoretical Physics, Universität Bonn, \\ Bonn D-53115, Germany \\ ${ }^{b}$ Skobeltsyn Institute of Nuclear Physics of Moscow State University, \\ Moscow 119992, Russian Federation \\ ${ }^{c}$ Moscow Center for Fundamental and Applied Mathematics, \\ Moscow 119992, Russian Federation \\ ${ }^{d}$ Rudolf Peierls Centre for Theoretical Physics, University of Oxford, \\ Clarendon Laboratory, Parks Road, Oxford OX1 3PU, U.K. \\ E-mail: cduhr@uni-bonn.de, smirnov@theory.sinp.msu.ru, \\ lorenzo.tancredi@cern.ch
}

ABSTRACT: We analytically evaluate the master integrals for the second type of planar contributions to the massive two-loop Bhabha scattering in QED using differential equations with canonical bases. We obtain results in terms of multiple polylogarithms for all the master integrals but one, for which we derive a compact result in terms of elliptic multiple polylogarithms. As a byproduct, we also provide a compact analytic result in terms of elliptic multiple polylogarithms for an integral belonging to the first family of planar Bhabha integrals, whose computation in terms of polylogarithms was addressed previously in the literature.

KEYwords: Scattering Amplitudes, Precision QED

ArXiv ePrint: 2108.03828 


\section{Contents}

1 Introduction 1

2 Canonical differential equations 3

3 From $d$ log-forms to multiple polylogarithms 5

$\begin{array}{lll}3.1 & \text { Rational alphabets without square roots } & 7\end{array}$

$\begin{array}{lll}3.2 & \text { Alphabets with a single elliptic square root } & 8\end{array}$

4 Integration of the differential equations in terms of MPLs 10

5 Compact analytic results in terms of eMPLs $\quad 14$

$\begin{array}{lll}5.1 \text { An analytic result for } f_{14} \text { in terms of eMPLs } & 14\end{array}$

$\begin{array}{ll}5.2 \text { A compact analytic result for the first planar family } & 18\end{array}$

6 Conclusions 20

$\begin{array}{ll}\text { A Canonical basis } & 21\end{array}$

$\begin{array}{ll}\text { B Elliptic multiple polylogarithms } & 23\end{array}$

\section{Introduction}

The Bhabha scattering process, i.e., the elastic scattering of an electron-positron pair, is one of the standard candles at lepton colliders, and it will play a crucial role at future circular or linear colliders. A precise theoretical knowledge of this process, including up to next-tonext-leading order effects in the QED coupling constant, is therefore highly desirable. So far the NNLO cross section is only known in the massless limit, supplemented by the non power-suppressed finite mass effects (see, e.g., ref. [1] for recent results). Complete NNLO results including the full dependence on the electron mass, however, are not yet available.

One of the main obstacles to obtain the complete NNLO results is the complexity of the two-loop integrals involved. The relevant two-loop integrals were evaluated in the smallmass limit in refs. [2-6]. Up to now, there are only partial results for two-loop integrals with massive fermions. Analytic results for diagrams with one-loop insertions and a closed massive fermion loop were obtained in refs. [7, 8]. First analytic results for massive twoloop double-box Bhabha diagrams were obtained in refs. $[9,10]$. More attempts to evaluate two-loop Bhabha integrals can be found in refs. [11-15].

The master integrals relevant for the calculation of the two-loop corrections to Bhabha scattering can be classified into two planar families and one non-planar one. The systematic evaluation of the integrals in the first family was started in ref. [16], where the master 


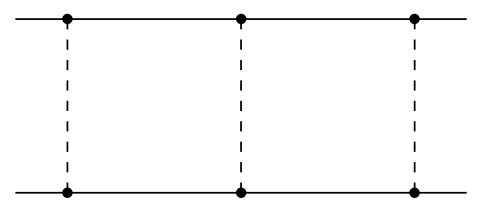

(a)

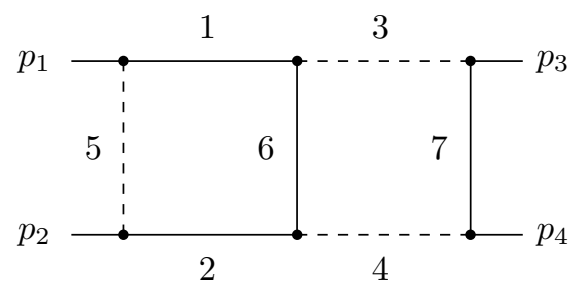

(b)

Figure 1. Planar graphs of the first and the second type for two-loop Bhabha scattering. Solid (dashed) lines indicate massive (massless) propagators. All the external momenta are incoming.

integrals for the family associated with graph (a) of figure 1 were evaluated in terms of multiple polylogarithms [17-19] (MPLs) in the framework of the method of differential equations [20-22] with the help of the strategy based on canonical bases [23]. In ref. [16], a solution in terms of MPLs had been provided for all integrals except one, whose analytic evaluation was hindered by the presence of the a non-rationalisable square-root in the symbol alphabet. More recently, it was shown that also this last integral can be expressed in terms of multiple polylogarithms by an integration technique based on an ansatz of MPLs with suitable arguments [24].

The goal of the present paper is to analytically evaluate the master integrals for the second planar family, which is associated with graph (b) of figure 1. In a way that is reminiscent of the first planar family of integrals, we will show that also in this case we can obtain results in terms of MPLs for all master integrals but one (see figure 2), due to the presence of the same non-rationalisable square root found in the evaluation of the master integrals for graph (a). While it can be shown by direct integration techniques that also for this master integral a representation in terms of MPLs exists, the representation we obtained is extremely cumbersome and of no practical use. Nevertheless, it turns out that a compact result for this integral can be derived in terms of the elliptic MPLs introduced in refs. [25-27]. As a byproduct of our analysis, we will also provide a very compact analytic result for the remaining master integral in the first family in figure 1(a) in terms of the same class of functions. ${ }^{1}$

The rest of the paper is organised as follows. In section 2 we explain the notation, present the system of canonical differential equations for the problem at hand, and introduce the alphabet needed to describe the planar family in figure 1(b). The alphabet contains multiple square roots, and it is a priori not obvious that the differential equations can be integrated in terms of multiple polylogarithms. In section 3 we review general sufficient criteria and algorithms to solve a system of canonical differential equations in terms of MPLs or their elliptic generalisations. We then continue in section 4 to apply these ideas to our computation. We explain how our differential equations can straightforwardly be solved in terms of multiple polylogarithms for all elements of the basis of the master integrals but one, which is connected with the graph shown in figure 2 . In section 5 we

\footnotetext{
${ }^{1}$ This result had first been presented by one of the authors at the Loops and Legs Conference 2018 in St. Goar, but it has never been published before.
} 


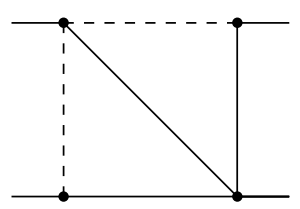

Figure 2. The graph associated with the master integral which we evaluate in terms of elliptic polylogarithms.

show how a convenient and compact representation for this integral can be found in terms of elliptic generalisations of MPLs. We also provide a very compact, alternative, analytic solution for one of the master integrals in the first planar family considered in refs. [23, 24]. Finally we draw our conclusions in section 6 .

\section{Canonical differential equations}

The Feynman integrals for the family of figure 1(b) can be organised in an integral family with nine propagators, where the first seven propagators correspond to the edges of the graph and the last two are so-called irreducible numerators,

$$
\begin{aligned}
& F_{a_{1}, a_{2}, \ldots, a_{9}}\left(s, t, m^{2} ; D\right) \\
& =\left(\frac{e^{\gamma_{E} \epsilon}}{i \pi^{D / 2}}\right)^{2} \int \frac{d^{D} k_{1} d^{D} k_{2}}{\left[-k_{1}^{2}+m^{2}\right]^{a_{1}}\left[-\left(k_{1}+p_{1}+p_{2}\right)^{2}+m^{2}\right]^{a_{2}}\left[-k_{2}^{2}\right]^{a_{3}}} \\
& \quad \times \frac{\left[-\left(k_{2}+p_{1}\right)^{2}\right]^{-a_{8}}\left[-\left(k_{1}-p_{3}\right)^{2}\right]^{-a_{9}}}{\left[-\left(k_{2}+p_{1}+p_{2}\right)^{2}\right]^{a_{4}}\left[-\left(k_{1}+p_{1}\right)^{2}\right]^{a_{5}}\left[-\left(k_{1}-k_{2}\right)^{2}+m^{2}\right]^{a_{6}}\left[-\left(k_{2}-p_{3}\right)^{2}+m^{2}\right]^{a_{7}}} .
\end{aligned}
$$

The indices $a_{i}, i=1 \ldots 7$ can be positive or negative integers, but we restrict our computation to $a_{8}, a_{9} \leq 0$. We work in dimensional regularisation in $D=4-2 \epsilon$ dimensions in order to regulate both infrared and ultraviolet divergences. The electron mass is denoted by $m$, and the external momenta $p_{i}$ are on shell, $p_{i}^{2}=m^{2}$. We introduce the usual Mandelstam variables

$$
s=\left(p_{1}+p_{2}\right)^{2}, \quad t=\left(p_{1}+p_{3}\right)^{2}, \quad u=\left(p_{2}+p_{3}\right)^{2},
$$

with $s+t+u=4 m^{2}$.

Using the public codes FIRE [28] and KIRA [29, 30], we solve the integration-by-parts (IBP) identities [31, 32] and reveal 43 independent master integrals, which we collect into the vector $g=\left(g_{1}, \ldots, g_{43}\right)^{T}$. This vector satisfies a system of linear differential equations of the form

$$
\partial_{v} g=A_{v} g \text {, }
$$

where $v=s, t, m^{2}, \partial_{v}=\frac{\partial}{\partial v}$ and the matrices $A_{s}, A_{t}, A_{m^{2}}$ are rational functions of $s, t, m^{2}$ and $\epsilon$. In the following, it will be useful to collect all the partial derivatives into a total differential, and to work with the equation

$$
d g=d A g, \quad d A=d s A_{s}+d t A_{t}+d m^{2} A_{m^{2}} .
$$


In order to evaluate the master integrals, it is convenient to search for a so-called canonical basis [23], i.e., a basis of master integrals for which the matrix $d A$ on the righthand side of eq. (2.4) is proportional to $\epsilon$ and its entries can all be expressed as linear combinations of total differentials of logarithms. While it has been suggested (at least conjecturally) that the study of the residues of the integrands can provide the full information to determine the elements of the canonical basis [33], this analysis can become computational very expensive when square-roots are involved. ${ }^{2}$ Therefore, we follow here a mixed approach to find a canonical basis. In order to select suitable candidates, we start by analysing only the leading singularities associated to the maximal cuts of the various integrals, and we supplement this analysis by the method of ref. [35]. By choosing integrals with unit leading singularities at the level of the maximal cuts, one can often bring the initial differential equations into a so-called precanonical form, where the corresponding matrices depend linearly on $\epsilon$. Once this is achieved, the prescriptions of ref. [35] can be successfully applied to arrive at a fully canonical form. In our case, the precanonical basis is composed of the 43 Feynman integrals present on the right-hand side of the above expression for the $\epsilon$-basis. In this way we obtain the new system of differential equations

$$
d f=\epsilon d \bar{A} f,
$$

with $\bar{A}$ independent of $\epsilon$. Our canonical basis $f_{i}$ is given in appendix A. The price to pay for casting the equations in canonical form is that the new matrix $d \bar{A}$ is not a matrix of rational one-forms, but it involves four square roots:

$$
\begin{aligned}
r_{s} & =\sqrt{-s} \sqrt{4 m^{2}-s}, & r_{t} & =\sqrt{-t} \sqrt{4 m^{2}-t}, \\
r_{u} & =\sqrt{-s-t} \sqrt{4 m^{2}-s-t}, & r_{s t} & =\sqrt{-s} \sqrt{4 m^{6}-s\left(m^{2}-t\right)^{2}} .
\end{aligned}
$$

More precisely, the matrix $\bar{A}$ takes the general form

$$
\bar{A}=\sum_{i=1}^{16} \bar{A}_{i} \log R_{i}\left(s, t, m^{2}\right),
$$

where the $R_{i}$ are algebraic functions, referred to as letters (and their collection is called the alphabet):

$$
\begin{aligned}
& R_{1}\left(s, t, m^{2}\right)=\frac{s}{m^{2}}, \\
& R_{2}\left(s, t, m^{2}\right)=\frac{s-4 m^{2}}{m^{2}}, \quad R_{3}\left(s, t, m^{2}\right)=\frac{s-r_{s}}{s+r_{s}}, \\
& R_{4}\left(s, t, m^{2}\right)=\frac{t}{m^{2}}, \\
& R_{5}\left(s, t, m^{2}\right)=\frac{t-4 m^{2}}{m^{2}}, \quad R_{6}\left(s, t, m^{2}\right)=\frac{t-r_{t}}{t+r_{t}}, \\
& R_{7}\left(s, t, m^{2}\right)=\frac{s+t}{m^{2}}, \\
& R_{8}\left(s, t, m^{2}\right)=\frac{s+t-4 m^{2}}{m^{2}}, \\
& R_{10}\left(s, t, m^{2}\right)=\frac{s t+r_{s} r_{t}}{s t-r_{s} r_{t}}, \\
& R_{11}\left(s, t, m^{2}\right)=\frac{(s+t) r_{s}-s r_{u}}{(s+t) r_{s}+s r_{u}} \\
& R_{12}\left(s, t, m^{2}\right)=\frac{(s+t) r_{t}-t r_{u}}{(s+t) r_{t}+t r_{u}}, \quad R_{13}\left(s, t, m^{2}\right)=\frac{r_{s t}-m^{2} s+s t}{r_{s t}+m^{2} s-s t}, \\
& R_{14}\left(s, t, m^{2}\right)=\frac{r_{t} r_{s t}-3 m^{2} s t+s t^{2}}{r_{t} r_{s t}+3 m^{2} s t-s t^{2}}, \quad R_{15}\left(s, t, m^{2}\right)=\frac{r_{s} r_{s t}+4 m^{4} s-m^{2} s^{2}+s^{2} t}{r_{s} r_{s t}-4 m^{4} s+m^{2} s^{2}-s^{2} t}, \\
& R_{16}\left(s, t, m^{2}\right)=\frac{m^{2} r_{s} r_{s t}-s r_{s} r_{s t}-4 m^{6} s-3 m^{4} s^{2}+m^{2} s^{3}+3 m^{2} s^{2} t-s^{3} t}{m^{2} r_{s} r_{s t}-s r_{s} r_{s t}+4 m^{6} s+3 m^{4} s^{2}-m^{2} s^{3}-3 m^{2} s^{2} t+s^{3} t} .
\end{aligned}
$$

\footnotetext{
${ }^{2}$ See ref. [34] for an automated implementation of this approach.
} 
As we will see, the appearance of these square roots makes the solution of the differential equation (2.5) in terms of MPLs highly non-trivial.

It is easy to write down a formal solution to eq. (2.5) as

$$
f\left(s, t, m^{2} ; \epsilon\right)=\mathbb{P} \exp \left[\epsilon \int_{\gamma} d \tilde{A}\right] f_{0}(\epsilon),
$$

where Pexp denotes the path-ordered exponential and $f_{0}(\epsilon)$ encodes the initial condition and is related to the value of $f$ at a specific point $\left(s_{0}, t_{0}, m_{0}^{2}\right)$. The path $\gamma$ connects the initial point $\left(s_{0}, t_{0}, m_{0}^{2}\right)$ to the generic point $\left(s, t, m^{2}\right)$.

When expanding eq. (2.9) in $\epsilon$, then at each order we can write $f$ in terms of Chen iterated integrals [36], defined in the following way: consider a path $\gamma$ and a collection of one-forms $\omega_{i}$. If $t$ denotes a local coordinate on $\gamma$, we can pull each $\omega_{i}$ back to $\gamma$ and write $\gamma^{*} \omega_{i}=d t F_{i}(t)$. We can then define the iterated integral of a sequence $\omega_{i_{1}} \ldots \omega_{i_{n}}$ (usually referred to as a word) by

$$
\int_{\gamma} \omega_{i_{1}} \ldots \omega_{i_{n}}=\int_{0 \leq t_{1} \leq \ldots \leq t_{n} \leq 1} d t_{n} F_{i_{n}}\left(t_{n}\right) \int_{0}^{t_{n}} \ldots \int_{0}^{t_{2}} d t_{1} F_{i_{1}}\left(t_{1}\right) .
$$

The number $n$ of integrations is called the length of the iterated integral. In general, this integral will not be homotopy-invariant, i.e., it will depend on the details of the path $\gamma$. There is a necessary and sufficient condition, called the integrability condition, for a combination of iterated integrals to be homotopy-invariant [36]. The details of this criterion are not important in the following. Here it suffices to say that it is always satisfied for the solutions in eq. (2.9). The corresponding Chen iterated integrals are then multi-valued functions of the end point $\left(s, t, m^{2}\right)$ of the path $\gamma$, where the multi-valuedness only comes from choosing two non-homotopic paths from $\left(s_{0}, t_{0}, m_{0}^{2}\right)$ to $\left(s, t, m^{2}\right)$.

The master integrals $f\left(s, t, m^{2} ; \epsilon\right)$ can be expressed at every order in terms of Chen iterated integrals over words from the alphabet in eq. (2.8). The coefficient of $\epsilon^{n}$ in the path-ordered exponential in eq. (2.9) only involves iterated integrals of length $n$. Chen iterated integrals are a very general class of functions, and it can be useful to express them in terms of a class of functions that are well-studied in the literature. In the next section we discuss sufficient criteria for when Chen iterated integrals over $d$ log-forms with algebraic arguments, as the ones above, can be expressed in terms of other classes of functions.

\section{From $d \log$-forms to multiple polylogarithms}

Since the solution of differential equations in canonical form as Laurent series in dimensional regularisation leads naturally to linear combinations of Chen iterated integrals over words of $d \log$-forms, it is natural to ask when it is possible to evaluate such iterated integrals in terms of other classes of functions, and if it is possible to perform this rewriting algorithmically. The advantage of this rewriting lies in the fact that these classes of functions may be well studied in the literature, and there may be established methods or computer codes for their manipulation and/or their numerical evaluation. 
Let us consider Chen iterated integrals of the form $\int_{\gamma} w$, where $\gamma$ is a path from an initial point $\mathbf{x}_{0}=\left(x_{0,1}, \ldots, x_{0, p}\right)$ to the point $\mathbf{x}_{1}=\left(x_{1,1}, \ldots, x_{1, p}\right)$, and $w$ is an integrable combination of words of length $n$ of the form

$$
w=\sum_{i_{1} \ldots i_{n}} c_{i_{1} \ldots i_{n}} d \log R_{i_{1}}(\mathbf{x}) \ldots d \log R_{i_{n}}(\mathbf{x}), \quad c_{i_{1} \ldots i_{n}} \in \mathbb{Q} .
$$

We consider the initial point $\mathbf{x}_{0}$ fixed, and we see the integral as a multi-valued function of the end-point $\mathbf{x}_{1}$. The arguments of the logarithms are assumed to be algebraic functions of the variables $\mathbf{x}=\left(x_{1}, \ldots, x_{p}\right)$.

For some time, there was a folklore belief in the physics community that all such iterated integrals could be evaluated in terms of a rather simple class of iterated integrals, namely the so-called multiple polylogarithms (MPLs), defined by [17-19, 37]

$$
G_{a_{1}, \ldots, a_{n}}(z)=G\left(a_{1}, \ldots, a_{n} ; z\right)=\int_{0}^{z} \frac{d t}{t-a_{1}} G\left(a_{2}, \ldots, a_{n} ; t\right),
$$

where the recursion starts with $G(; z) \equiv 1$. In the special case where all the $a_{i}$ 's are zero, we define

$$
G_{0, \ldots, 0}(z)=G(\underbrace{0, \ldots, 0}_{n} ; z)=\frac{1}{n !} \log ^{n} z .
$$

The shared belief in physics was that, whenever the $d$ log's in eq. (3.1) have algebraic arguments $R_{i}$, then this integral can be written in terms of MPLs whose arguments $a_{i}$ and $z$ are algebraic functions of $\mathbf{x}_{0}$ and $\mathbf{x}_{1}$. This belief was shown to be false in ref. [38], where an example of an iterated integral of length two was constructed that cannot be evaluated in terms of MPLs with algebraic arguments. The result of ref. [38] shows that the question of whether an iterated integral of $d \log$-forms with algebraic arguments can be expressed in terms of MPLs depends in general on the details of the integral, including the details of the integration contour.

In the remainder of this section we discuss some special cases of algebraic letters $R_{i}$ where the Chen iterated integrals can be evaluated in terms of other classes of special functions. The starting point is the observation that in the context of Feynman integrals, the $R_{i}$ are not generic algebraic functions, but often involve at most square roots of polynomials, i.e., they are of the form:

$$
R_{i}(\mathbf{x})=r_{i, 0}(\mathbf{x})+\sum_{a=1}^{N_{\mathrm{sqrt}}} r_{i, a}(\mathbf{x}) \sqrt{q_{a}(\mathbf{x})},
$$

where the $r_{i, j}$ are rational functions, and the $q_{a}$ are polynomials. Without loss of generality, we assume the $r_{i, j}$ to be polynomials. At this point we have to make a comment: the integrand of the iterated integration, and thus the functional form of the letters $R_{i}$, is sensitive to a change of variables. In particular, one may ask if we can find a change of variables $\mathbf{x}=\psi(\mathbf{y})$, with $\psi$ a rational function, such that $q_{a}(\psi(\mathbf{y}))=u_{a}(\mathbf{y})^{2}$ is a perfect square, for some values of $a$. This operation is known as rationalisation of square roots. Over the last few years, several algorithmic criteria have been developed to find such a parametrisation for specific Feynman integral computations, or to prove that rationalisation 
is instead not possible [39-44]. Note that rationalisability can easily be decided for a single square root of a one-variable polynomial $\left(p=N_{\mathrm{sqrt}}=1\right)$ based on the degree $\operatorname{deg} q_{1}$ of the polynomial: the square root $\sqrt{q_{1}\left(x_{1}\right)}$ can be rationalised (and the corresponding function $\psi$ can be constructed explicitly) if and only if $\operatorname{deg} q_{1} \leq 2$. The question of the rationalisability for $p>1$ is much more involved, even in the presence of a single square root, see refs. [40-44].

In the following we discuss two special cases of eq. (3.4), in which we can express the Chen iterated integrals in terms of other classes of iterated integrals:

- If $N_{\mathrm{sqrt}}=0$, the Chen iterated integrals can be expressed in terms of MPLs evaluated at algebraic arguments.

- If $N_{\text {sqrt }}=1$ and $\operatorname{deg} q_{1}=3$ or 4 , the Chen iterated integrals can be expressed in terms of elliptic generalisations of MPLs evaluated at algebraic arguments.

In particular, we explain how we can algorithmically rewrite all Chen iterated integrals that meet these criteria in terms of MPLs and their elliptic analogues. This algorithm is well known, but we document it here because it will be an important tool to obtain compact analytic expressions for some master integrals that contribute to Bhabha scattering at two loops. Two comments are in order: first, in the presence of (multiple) square roots, it may be possible to change variables and rationalise (some of) the roots. In this way it may be possible to reduce the problem to a situation covered by the criteria above, even though the original problem did not satisfy these conditions. Second, we stress that the aforementioned conditions are only sufficient, but by no means necessary, to rewrite Chen iterated integrals in terms of (elliptic) MPLs. In particular, there are several examples of Feynman integrals whose alphabets involve non-rationalisable square roots, but nevertheless, it was possible to express them in terms of ordinary MPLs, cf., e.g., refs. [24, 45, 46].

\subsection{Rational alphabets without square roots}

If the alphabet does not contain any square roots, or if all square roots can be rationalised, we can always express the Chen iterated integral in terms of MPLs evaluated at algebraic arguments. First, we can use the additivity of the logarithm to assume that all letters $R_{i}$ are irreducible polynomials. Next, we can use the homotopy-invariance of the integral to deform the contour $\gamma$ into a new contour $\gamma_{0}$ with the same end-points $\mathbf{x}_{0}$ and $\mathbf{x}_{1}$, without changing the value of the integral (except for picking up residues when we cross a pole). We choose the new contour as follows: we order the integration variables in some way, which for simplicity we assume to be the natural order $x_{1}, \ldots, x_{p}$. The contour $\gamma_{0}$ is then obtained as the concatenation of the straight-line segments $\gamma_{r}, 1 \leq r \leq p$, defined by $\mathbf{x}=\varphi^{(r)}(t)=\left(\varphi_{1}^{(r)}(t) \ldots, \varphi_{p}^{(r)}(t)\right)$ with $0 \leq t \leq 1$ and

$$
\varphi_{i}^{(r)}(t)= \begin{cases}x_{1, i}, & i<r \\ \left(x_{1, r}-x_{0, r}\right) t+x_{0, r}, & i=r \\ x_{0, i}, & i>r .\end{cases}
$$


Next we can iteratively apply the path-composition formula for iterated integrals,

$$
\int_{\gamma_{1} \gamma_{2}} \omega_{i_{1}} \ldots \omega_{i_{n}}=\sum_{k=0}^{n} \int_{\gamma_{1}} \omega_{i_{1}} \ldots \omega_{i_{k}} \int_{\gamma_{2}} \omega_{i_{k+1}} \ldots \omega_{i_{n}}, \quad \omega_{i}=d \log R_{i}(\mathbf{x}) .
$$

The previous equation allows us to reduce the integral to a linear combination of products of Chen iterated integrals over the straight-line segments $\gamma_{r}$. On the line segment $\gamma_{r}$ the $d \log$-forms take a particularly simple form. Indeed, since all the letters $R_{i}$ are polynomials in $\mathbf{x}$, it is easy to see that $R_{i}\left(\varphi_{r}(t)\right)$ is a polynomial in $t$. In the following we write

$$
R_{i}\left(\varphi_{r}(t)\right)=c_{0}\left(t-c_{1}\right) \ldots\left(t-c_{d}\right)
$$

where $d$ denotes the degree of the polynomial $R_{i}\left(\varphi_{r}(t)\right)$ and the quantities $c_{j}, 0 \leq j \leq d$ are algebraic functions of $\mathbf{x}_{0}$ and $\mathbf{x}_{1}$. The pull-back of the one-form $d \log R_{i}(\mathbf{x})$ to the straight-line segment $\gamma_{r}$ then reads

$$
\gamma_{r}^{*} d \log R_{i}(\mathbf{x})=d t F_{r, i}(t)
$$

with

$$
F_{r, i}(t)= \begin{cases}0, & \text { if } \partial_{x_{r}} R_{i}(\mathbf{x})=0 \\ \sum_{j=1}^{d} \frac{1}{t-c_{j}}, & \text { if } \partial_{x_{r}} R_{i}(\mathbf{x}) \neq 0\end{cases}
$$

The previous equation makes it manifest that on each straight-line segment the Chen iterated integral evaluates to ordinary MPLs with algebraic arguments $c_{j}$, cf. eq. (3.2).

\subsection{Alphabets with a single elliptic square root}

We have already seen that a square root of a polynomial of degree three or four cannot be rationalised. More precisely, consider the set of points $(x, y)$ constrained by

$$
y^{2}=P_{n}(x)
$$

where $P_{n}(x)$ is a polynomial of degree $n$ in $x$. If $n=3$ or 4 , this equation defines an algebraic variety called an elliptic curve. It is thus not surprising that the case $N_{\text {sqrt }}=1$ and $q_{1}=P_{n}$, with $n=3$ or 4 , leads to generalisations of MPLs related to elliptic curves. We start by giving a lightning review of elliptic multiple polylogarithms (eMPLs), before we comment on the generalisation of the algorithm from section 3.1. More details can be found in appendix B.

We focus here on the case $n=4$, and we assume that $P_{4}$ has the form $P_{4}(x)=$ $\left(x-a_{1}\right)\left(x-a_{2}\right)\left(x-a_{3}\right)\left(x-a_{4}\right)$. Elliptic multiple polylogarithms (eMPLs) can then be defined as the iterated integrals [25, 47-49]

$$
\mathcal{E}_{4}\left(\begin{array}{cccc}
n_{1} & \ldots & n_{k} \\
c_{1} & \ldots & c_{k}
\end{array} ; x, \vec{a}\right)=\int_{0}^{x} d t \Psi_{n_{1}}\left(c_{1}, t, \vec{a}\right) \mathcal{E}_{4}\left(\begin{array}{cccc}
n_{2} & \ldots & n_{k} \\
c_{2} & \ldots & c_{k}
\end{array} ; t, \vec{a}\right),
$$

with $n_{i} \in \mathbb{Z}$ and $c_{i} \in \mathbb{C} \cup\{\infty\}$, and $\vec{a}=\left(a_{1}, a_{2}, a_{3}, a_{4}\right)$ is the vector of the four branch points $a_{i}$. Just like ordinary MPLs, eMPLs have at most logarithmic singularities, but no poles. The number $n=\sum_{i=1}^{k}\left|n_{i}\right|$ is called the weight of the eMPL, and the number of 
integrations $k$ is its length. In the case where all the indices $A_{i}=\left(\begin{array}{c}n_{i} \\ c_{i}\end{array}\right)$ are equal to $\left(\begin{array}{c} \pm 1 \\ 0\end{array}\right)$, the integral is divergent and requires a special treatment similar to the case $a_{n}=0$ for ordinary MPLs, cf. eq. (3.3). We refer to appendix B for details. Also note that we need to be careful about how we choose the branches of the square root $y=\sqrt{P_{4}(x)}$. We will come back to this point in section 5 .

There are infinitely many integration kernels $\Psi_{n}(c, x, \vec{a})$ for given $(c, x, \vec{a})$ in eq. (3.11). In concrete applications only a finite number of these kernels appear. Here we only list the kernels with $|n| \leq 1$, which are of direct relevance to this paper. For $n=0$, we have

$$
\Psi_{0}(0, x, \vec{a})=\frac{c_{4}}{\omega_{1} y},
$$

with $c_{4}=\frac{1}{2} \sqrt{a_{13} a_{24}}, a_{i j}=a_{i}-a_{j} . \omega_{1}$ is one of the two periods associated to the elliptic curve defined by the equation $y^{2}=P_{4}(x)$,

$$
\omega_{1}=2 \mathrm{~K}(\lambda), \quad \omega_{2}=2 i \mathrm{~K}(1-\lambda), \quad \lambda=\frac{a_{14} a_{23}}{a_{13} a_{24}},
$$

where $\mathrm{K}$ is the complete elliptic integral of the first kind

$$
\mathrm{K}(\lambda)=\int_{0}^{1} \frac{d t}{\sqrt{\left(1-t^{2}\right)\left(1-\lambda t^{2}\right)}} .
$$

For $|n|=1$, we have (with $c \neq \infty$ )

$$
\begin{aligned}
\Psi_{1}(c, x, \vec{a}) & =\frac{1}{x-c}, \\
\Psi_{-1}(c, x, \vec{a}) & =\frac{y_{c}}{y(x-c)}+Z_{4}(c, \vec{a}) \frac{c_{4}}{y}, \\
\Psi_{1}(\infty, x, \vec{a}) & =-Z_{4}(x, \vec{a}) \frac{c_{4}}{y}, \\
\Psi_{-1}(\infty, x, \vec{a}) & =\frac{x}{y}-\frac{1}{y}\left[a_{1}+2 c_{4} G_{*}(\vec{a})\right],
\end{aligned}
$$

where we introduced the shorthand $y_{c}=\sqrt{P_{4}(c)}$. These functions have the property that the differential form $d x \Psi_{ \pm 1}(c, x, \vec{a})$ has a simple pole at $x=c$, and no other poles (except for $d x \Psi_{1}(c, x, \vec{a})$, which always has a pole at $\left.x=\infty\right)$. Note that the kernel $\Psi_{1}$ is identical to the kernel that defines ordinary MPLs (cf. eq. (3.2)), and so ordinary MPLs are a subset of eMPLs,

$$
\mathcal{E}_{4}\left(\begin{array}{ccc}
1 & \ldots & 1 \\
c_{1} & \ldots & c_{k}
\end{array} ; x, \vec{a}\right)=G\left(c_{1}, \ldots, c_{k} ; x\right) .
$$

The functions $Z_{4}(c, \vec{a})$ and $G_{*}(\vec{a})$ in eq. (3.15) are in general transcendental functions. They can be expressed in terms of incomplete elliptic integrals of the first and second kind (see appendix B). Depending on the value of the argument $c$, in many applications it is possible to evaluate $Z_{4}(c, \vec{a})$ and $G_{*}(\vec{a})$ in the form $A+B \frac{i \pi}{\omega_{1}}$, where $A$ and $B$ are algebraic functions of $\vec{a}$ and $c$ [49]. In particular, in all cases relevant for this paper, $Z_{4}(c, \vec{a})$ and $G_{*}(\vec{a})$ will always be algebraic functions (see section 5.1).

Let us now assume that the alphabet is given by $d \log$-forms with arguments

$$
R_{i}(\mathbf{x})=r_{i, 0}(\mathbf{x})+r_{i, 1}(\mathbf{x}) \sqrt{q_{1}(\mathbf{x})},
$$


where $r_{i, 0}(\mathbf{x})$ are polynomials in $\mathbf{x}$ and $q_{1}(\mathbf{x})$ is a non-constant polynomial of degree at most four. We assume that the $r_{i, j}(\mathbf{x})$ do not have any common zero (otherwise we could factor out a term from this letter in the alphabet), and $q_{1}(\mathbf{x})$ does not have a double zero (otherwise we could factor it out of the square root). We allow $r_{i, 1}(\mathbf{x})$ to be zero (in which case this letter does not depend on the square root), but $r_{i, 0}(\mathbf{x})$ is assumed not to vanish. We can separate all the letters into even and odd parts:

$$
d \log R_{i}(\mathbf{x})=\frac{1}{2} d \log R_{i}^{+}(\mathbf{x})-\frac{1}{2} d \log R_{i}^{-}(\mathbf{x}),
$$

with

$$
\begin{aligned}
& R_{i}^{+}(\mathbf{x})=r_{i, 0}(\mathbf{x})^{2}-r_{i, 1}(\mathbf{x})^{2} q_{1}(\mathbf{x}) \\
& R_{i}^{-}(\mathbf{x})=\frac{r_{i, 0}(\mathbf{x})-r_{i, 1}(\mathbf{x}) \sqrt{q_{1}(\mathbf{x})}}{r_{i, 0}(\mathbf{x})+r_{i, 1}(\mathbf{x}) \sqrt{q_{1}(\mathbf{x})}} .
\end{aligned}
$$

Next we deform the integration path $\gamma$ to the sequence of line segments $\gamma_{r}$ defined in eq. (3.5). Even letters give rise to integration kernels of the form $\frac{d t}{t-c_{j}}$ and can be dealt with exactly as in section 3.1. We will not discuss them any further. For the odd letters, note that on the straight-line segment $\gamma_{r}, q_{1}\left(\varphi_{r}(t)\right)$ is a polynomial of degree $n$ in $t$, with $n \leq 4$. We write:

$$
q_{1}\left(\varphi_{r}(t)\right)=\alpha_{r} P^{(r)}(t)=\alpha_{r}\left(t-a_{r, 1}\right) \cdots\left(t-a_{r, n}\right), \quad \vec{a}_{r}=\left(a_{r, 1}, \ldots, a_{r, n}\right) .
$$

If $n \leq 2$, then the square root $\sqrt{P^{(r)}(t)}$ can be rationalised, and all letters are rational on the line segment $\gamma_{r}$, and we do not need to discuss this case anymore. If the degree of $P^{(r)}(t)$ is three or four, then we cannot rationalise the square root on $\gamma_{r}$. Instead, we obtain

$$
\gamma_{r}^{*} d \log R_{i}^{-}\left(\varphi_{r}(t)\right)=d t F_{r, i}^{-}(t),
$$

where the parity of $R_{i}^{-}$implies that $F_{r, i}^{-}(t)$ is of the form

$$
F_{r, i}^{-}(t)=\frac{1}{\sqrt{P^{(r)}(t)}} S_{r, i}(t),
$$

where $S_{r, i}(t)$ is a rational function in $t$. At this point we make an important observation: by construction, the differential form $d \log R_{i}^{-}\left(\varphi_{r}(t)\right)$ has only logarithmic singularities. This implies that $S_{r, i}(t)$ has poles of order at most one (possibly including a simple pole at infinity). As a consequence, $S_{r, i}(t)$ may be written as a linear combination of the functions $\Psi_{-1}\left(c_{j}, t, \vec{a}_{r}\right)$ and $\Psi_{0}\left(c_{j}, t, \vec{a}_{r}\right)$. Hence, we can evaluate the integral on $\gamma_{r}$ in terms of eMPLs for the elliptic curve associated to $P^{(r)}(t)$. Note that, a priori, we may obtain eMPLs with different values of $\vec{a}_{r}$ for each segment $\gamma_{r}$.

\section{Integration of the differential equations in terms of MPLs}

After the general remarks of the previous section, we go back to the explicit solution for the system in eq. (2.5). The standard way to rationalize the first two square roots in eq. (2.6) is 
to turn to the dimensionless Landau variables $x$ and $y$ related to the Mandelstam invariants by

$$
\frac{-s}{m^{2}}=\frac{(1-x)^{2}}{x} \text { and } \frac{-t}{m^{2}}=\frac{(1-y)^{2}}{y} .
$$

In terms of these variables the Euclidean region $s, t<0$ corresponds to $0 \leq x, y \leq 1$ (using the symmetry of eq. (4.1) under $(x, y) \rightarrow(1 / x, 1 / y))$.

There exist different strategies to solve the differential equation in eq. (2.5). The first method consists in evaluating numerically the $\epsilon$-expansion of the path-ordered exponential in eq. (2.9). This can be achieved by application of the Frobenius method to look for powerseries solutions of ordinary differential equations in the vicinity of regular singular points. The Frobenius method has been successfully used in various one-dimensional problems in the past [50-53], and more recently a generalisation of this method has been proposed in ref. [54] to deal with complicated multidimensional problems, see for example [55, 56]. This strategy has also been implemented into the public code DiffExp [57]. ${ }^{3}$ The input data for this code are the matrices that define the differential equations, and the boundary conditions in some limit, e.g., at some point $\left(x_{0}, y_{0}\right)$. The code then uses this input to evolve the solution numerically from the point $\left(x_{0}, y_{0}\right)$ to some generic point $(x, y)$. We fix the boundary conditions in the limit $s, t \rightarrow 0$, which corresponds to $\left(x_{0}, y_{0}\right)=(1,1)$ in terms of the Landau variables in eq. (4.1). Using the expansion by regions strategy implemented in the public code asy.m [58, 59] (which is now included in the code FIESTA [60]) we obtain the following leading order asymptotic behaviour in this limit

$$
\begin{aligned}
& f_{1} \sim 1+\frac{\pi^{2} \epsilon^{2}}{6}-\frac{2 \zeta_{3} \epsilon^{3}}{3}+\frac{7 \pi^{4} \epsilon^{4}}{360}+\mathcal{O}\left(\epsilon^{5}\right) \\
& f_{6} \sim-\frac{1}{4}-\frac{5 \pi^{2} \epsilon^{2}}{24}-\frac{11 \zeta_{3} \epsilon^{3}}{6}-\frac{101}{480} \pi^{4} \epsilon^{4}+\mathcal{O}\left(\epsilon^{5}\right) \\
& f_{9} \sim-\frac{\pi^{2} \epsilon^{2}}{12}+\frac{1}{4} \epsilon^{3}\left(2 \pi^{2} \log 2-7 \zeta_{3}\right) \\
&+\frac{1}{180} \epsilon^{4}\left(13 \pi^{4}-90 \log ^{4} 2-180 \pi^{2} \log ^{2} 2-2160 \operatorname{Li}_{4}\left(\frac{1}{2}\right)\right)+\mathcal{O}\left(\epsilon^{5}\right) \\
& f_{18} \sim \frac{1}{2} \epsilon^{3}\left(2 \pi^{2} \log 2-3 \zeta_{3}\right)+\frac{1}{20} \epsilon^{4}\left(7 \pi^{4}-20 \log ^{4} 2-40 \pi^{2} \log ^{2} 2-480 \operatorname{Li}_{4}\left(\frac{1}{2}\right)\right)+\mathcal{O}\left(\epsilon^{5}\right), \\
& f_{19} \sim(-s)^{-\epsilon}\left(-1+\frac{8 \zeta_{3} \epsilon^{3}}{3}+\frac{\pi^{4} \epsilon^{4}}{30}\right)+\mathcal{O}\left(\epsilon^{5}\right) \\
& f_{22} \sim(-s)^{-\epsilon}\left(-\frac{1}{2}+\frac{4 \zeta_{3} \epsilon^{3}}{3}+\frac{\pi^{4} \epsilon^{4}}{60}\right)+(-s)^{-2 \epsilon}\left(\frac{1}{4}-\frac{\pi^{2} \epsilon^{2}}{24}-\frac{14 \zeta_{3} \epsilon^{3}}{3}-\frac{67}{480} \pi^{4} \epsilon^{4}\right)+\mathcal{O}\left(\epsilon^{5}\right) \\
& f_{23} \sim(-s)^{-2 \epsilon}\left(\pi^{2} \epsilon^{2}+2 \pi^{2} \epsilon^{3} \log 2+2 \pi^{2} \epsilon^{4}\left(\pi^{2}+\log ^{2} 2\right)\right)+\mathcal{O}\left(\epsilon^{5}\right) \\
& f_{25} \sim(-s)^{-\epsilon}\left(-\pi^{2} \epsilon^{2}-2 \pi^{2} \epsilon^{3} \log 2-\frac{1}{2} \pi^{2} \epsilon^{4}\left(\pi^{2}+4 \log ^{2} 2\right)\right)+\mathcal{O}\left(\epsilon^{5}\right)
\end{aligned}
$$

and $f_{i} \sim 0$, i.e., $f_{i}=\mathcal{O}(s, t)$, for all the other elements.

Let us emphasise that, in order to profit maximally from the automated code DiffExp, it is crucial that the input data are in an optimal form. This includes providing differential

\footnotetext{
${ }^{3} \mathrm{~A}$ Mathematica notebook is provided as supplementary material attached to this paper.
} 
equations in canonical form and fixing the boundary conditions in a simple point ( $s=t=0$, i.e., $x=y=1$ ). With our input, the code works very well and provides the possibility to obtain high-precision numerical results (100 digits accuracy and more), both in the Euclidean and the physical regions. In other words, DiffExp allows us to obtain highprecision numerical results for all master integrals and for all values of the input parameters. The code runs fast enough to allow its usage for practical applications. This is then of course sufficient for all phenomenological applications one has in mind. However, both from a formal and from a practical point of view, it may still be desirable in some situations to have full-fledged analytic representations of the master integrals in terms of functions that are well studied in the literature, e.g., MPLs. In the remainder of this section we explain how we can obtain analytic results for all master integrals but $f_{14}$ in terms of MPLs evaluated at algebraic arguments. The reason why $f_{14}$ is different will be discussed in section 5. Here it suffices to say that the square root $r_{u}$ only enters the differential equation for $f_{14}$. Since our strategy of solving the differential equations in terms of MPLs will be closely based on the ideas from section 3.1, it is not suprising that an additional square root implies that the sufficient condition of section 3.1 is not satisfied. We will discuss the case of $f_{14}$ in detail in section 5 , and we focus for the rest of this section only on the other master integrals.

In order to solve the master integrals in terms of MPLs, we start by observing that the square root $r_{s t}$ does not appear when solving the differential equations up to weight 3 for all elements but $f_{37}$, and at weight 4 for all elements but $f_{i}, i \in\{35,36,37,38,39,41,43\}$. The equations can be solved by integrating first in $x$, resulting in MPLs of the form $G(\vec{c} ; x)$ with $c_{i} \in\{0,-1,1,-y,-1 / y\}$. This allows us to fix the solution up to a function of $y$, which can be determined by substituting the results of the $x$-integration into the differential equations in $y$, checking that the variable $x$ disappears in them, and finally solving them in terms of MPLs of the form $G(\vec{c} ; y)$ with $c_{i} \in\{0,-1,1\}$, i.e., harmonic polylogarithms [37]. At this point the solution is fixed up to a set of undetermined integration constants, which we fix using the boundary conditions in eq. (4.2).

To evaluate $f_{37}$ at weights 3 and 4 and $f_{i}$ with $i \in\{35,36,38,39,41,43\}$ at weight 4 , we have to deal with the square root $r_{s t}$. It can be rationalized by the following further change of variables

$$
x=2 \frac{(1-w)\left(y^{2}-y+1\right)^{2}-2 y^{2}}{\left(1-w^{2}\right)\left(y^{2}-y+1\right)^{2}} .
$$

The resulting system of differential equations for 42 elements can be solved, first in $w$ and then in $y$. Solving in $w$ gives a linear combination of MPLs $G(\ldots ; w)$ with the letters the alphabet $\left\{l_{1}^{w}, \ldots, l_{15}^{w}\right\}$, where $l_{i}^{w}, i=1, \ldots, 11$, are taken from the set

$$
\begin{aligned}
\left\{1,-1, \frac{y^{4}-2 y^{3}+y^{2}-2 y+1}{\left(y^{2}-y+1\right)^{2}}, \frac{y^{2}+y+1}{y^{2}-y+1}, \frac{y^{2}-3 y+1}{y^{2}-y+1},\right. \\
-\frac{y^{2}+2 \sqrt{y^{2}+1} y-2 \sqrt{y^{2}+1}-y+1}{y^{2}-y+1},-\frac{y^{2}-2 \sqrt{y^{2}+1} y+2 \sqrt{y^{2}+1}-y+1}{y^{2}-y+1}, \\
\left.\quad \frac{y^{2}-y-1}{y^{2}-y+1},-\frac{y^{2}+y-1}{y^{2}-y+1},-\frac{2 y^{3}-y^{2}+y-1}{y^{2}-y+1}, \frac{y^{3}-y^{2}+y-2}{y\left(y^{2}-y+1\right)}\right\},
\end{aligned}
$$


and $l_{i}^{w}, i=12, \ldots, 15$ are the roots of the polynomial $\mathcal{Q}(y, w)$ defined by

$$
\begin{aligned}
\mathcal{Q}(y, w)= & w^{4}\left(y^{2}-y+1\right)^{4} y+2 w^{3}\left(y^{2}-4 y+1\right)\left(y^{2}-y+1\right)^{4} \\
& -2 w^{2}\left(y^{2}-y+1\right)^{2}\left(y^{6}-7 y^{5}+12 y^{4}-11 y^{3}+12 y^{2}-7 y+1\right) \\
& -2 w\left(y^{2}-y+1\right)^{2}\left(y^{6}-2 y^{5}+4 y^{4}-12 y^{3}+4 y^{2}-2 y+1\right) \\
& +2 y^{10}-11 y^{9}+30 y^{8}-62 y^{7}+90 y^{6}-89 y^{5}+90 y^{4}-62 y^{3}+30 y^{2}-11 y+2 .
\end{aligned}
$$

Solving the differential equation in $y$ (where the dependence on $w$ drops out) gives a linear combination of MPLs $G(\ldots ; y)$ with the letters from the set $\left\{l_{1}^{y}, \ldots, 23\right\}$, with

$$
\begin{aligned}
& l_{1}^{y}=0, l_{6}^{y}=\frac{1}{2}(3-\sqrt{5}), l_{7}^{y}=\frac{1}{2}(3+\sqrt{5}), l_{8}^{y}=e^{\frac{i \pi}{3}}, l_{9}^{y}=e^{-\frac{i \pi}{3}}, l_{10}^{y}=-e^{\frac{i \pi}{3}}, \\
& l_{11}^{y}=-e^{-\frac{i \pi}{3}}, l_{12}^{y}=1, l_{13}^{y}=-1, l_{24}^{y}=\frac{1}{2}(1-\sqrt{5}), l_{25}^{y}=\frac{1}{2}(1+\sqrt{5}), \\
& l_{26}^{y}=\frac{1}{2}(-1-\sqrt{5}), l_{27}^{y}=\frac{1}{2}(\sqrt{5}-1) .
\end{aligned}
$$

Moreover, $l_{i}^{y}, i=2, \ldots, 5$ are the roots of $1-2 y+y^{2}-2 y^{3}+y^{4} ; l_{i}^{y}, i=14, \ldots, 17$ are the roots of $3-6 y+5 y^{2}-6 y^{3}+3 y^{4} ; l_{i}^{y}, i=18, \ldots, 20$ are the roots of $-2+y-y^{2}+y^{3}$ and $l_{i}^{y}, i=21, \ldots, 23$ are the roots of $-1+y-y^{2}+2 y^{3}$.

Following this procedure, we obtain complete analytical results for $f_{37}$ at weights 3 and 4 , and for the elements $f_{i}$ with $i \in\{35,36,38,39,41,43\}$ at weight 4 , in terms of MPLs in $w$ or $y$ with the letters defined above. We have checked our analytic results for the master integrals against FIESTA [60] using also the forthcoming new release [61]. It turns out, however, (as it is easy to imagine from the dimension of the alphabet) that our results at weight 4 for these elements are rather complicated due to a very intricate branch-cut structure. In particular, evaluating them in this form at a phase-space point with GiNaC $[62,63]$ meets problems connected both with timing and stability, so that our results for the complicated elements become impractical, and for phenomenological applications the numerical solution obtained using DiffExp might be preferable. For the same reason, we also recommend to turn to DiffExp also for the contribution of element 37 of weight 3 . Of course, this does not imply that a better analytical representation in terms of MPLs does not exist, whose numerical evaluation could be much faster than automated numerical codes. Our analytic results obtained by direct integration of the differential equations could be used as a starting point to look for such a better analytic representation, if required.

Since we have complete analytic results in terms of MPLs through weight three, our results are sufficient to express the ultraviolet and infrared poles of the amplitude in terms of MPLs, and to check the cancellation of the poles analytically. Moreover, the analytic results at weight four may be useful in the future to study certain limits of the amplitude analytically, e.g., the small mass limit $m \ll 1$ or the high-energy limit $s \gg 1$.

Let us observe that, for the simpler master integrals, namely the ones which can be expressed as MPLs of the variables $(x, y)$, the analytic evaluation of the results in terms of MPLs in the physical region can be performed with GiNaC by adding small imaginary 
parts to $s$ and $t$. When applying DiffExp, the analytic continuation in the parametric integrals over segments of lines is performed by assigning an imaginary part of the form $\pm i \delta$ to the line parameter, in accordance with the Feynman prescription. In the code, such a choice is fixed by the option DeltaPrescriptions. In particular, in the supplementary file attached to this paper, the choice we make is DeltaPrescriptions $\rightarrow 4 \mathrm{~m} 2$ - s - I Delta, -s - I Delta,.... We observe that for these integrals the performance of GiNaC and DiffExp is more or less the same. For example, the evaluation of all the elements of the canonical basis with the accuracy of 100 digits takes several minutes with both codes. For the more complicated master integrals, which cannot be expressed in terms of MPLs with arguments that are rational in $(x, y)$, the performance of DiffExp stays the same, but GiNaC is much slower. This feature shows that the implementation of the method of power series expansions, with matching of neighbouring domains, in DiffExp is very successful. We note, however, that in the Euclidean region the evaluation with GiNaC is one order of magnitude faster, while the evaluation time for DiffExp is roughly the same. In the Euclidean region, our analytic expressions are thus preferable over DiffExp. It would be interesting to obtain an expression in the physical region where all MPLs admit a fast-converging Taylor series that may lead to a similar speed-up as in the Euclidean region.

\section{Compact analytic results in terms of eMPLs}

\subsection{An analytic result for $f_{14}$ in terms of eMPLs}

As mentioned in the previous section, we could solve the differential equations for all master integrals analytically in terms of MPLs with algebraic arguments, except for $f_{14}$. The reason why we cannot easily apply the techniques from the previous section to $f_{14}$ can be seen from the form of the differential equation itself. If we write $f_{14}(x, y)=\epsilon^{4} \bar{f}(x, y)$, then the differential equation in $x$ takes the form:

$$
\begin{aligned}
\frac{\partial}{\partial x} \bar{f}(x, y)= & \frac{1}{(x-1) x \sqrt{\Delta(x, y)}}\left\{-2(x+1) x\left(y^{2}-1\right) G_{0}(y) G_{0,0}(x)\right. \\
& (x-1) G_{0}(x)\left[2\left(3 x^{2} y+x(y-1)^{2}+y\right) G_{0,0}(y)+\pi^{2}\left(x^{2}-1\right) y\right] \\
- & (x+1)(x-1)^{2} y\left[2 G_{0}(y)\left(G_{-1 / y, 0}(x)-G_{-y, 0}(x)\right)+2 G_{-1 / y, 0,0}(x)+2 G_{-y, 0,0}(x)\right. \\
+ & 2 G_{0,0,0}(x)+4 G_{1,0,0}(x)-4 G_{0,0,0}(y)+4 G_{1,0,0}(y)+2 \zeta_{3}+\left(2 G_{0,0}(y)+\pi^{2}\right) G_{-1 / y}(x) \\
+ & \left.\left.\left(2 G_{0,0}(y)+\pi^{2}\right) G_{-y}(x)\right]\right\},
\end{aligned}
$$

with

$$
\Delta(x, y)=(x+y)(x y+1)\left(x^{2} y+x y^{2}-4 x y+x+y\right) .
$$

Due to the symmetry $f_{14}(x, y)=f_{14}(y, x)$, the differential equation with respect to $y$ can easily be obtained by exchanging the roles of $x$ and $y$ in eq. (5.1). The square root in eq. (5.1) is identical to the square root that has appeared in ref. [16] in the computation of the first planar two-loop family for Bhabha scattering. The polynomial under the square 
root has degree four in both $x$ and $y$, so it cannot be rationalised in one variable only. However, this is not sufficient to exclude that one cannot rationalise it by performing a change of variable involving simultaneously $x$ and $y$. In ref. [39] it was shown that the algebraic variety defined by $\xi^{2}=\Delta(x, y)$ is a K3 surface. As a consequence, it cannot be rationalised by any rational change of variables, and the strategy of rationalising all square roots and evaluating all iterated integrals in terms of MPLs using ideas from section 3.1 cannot be applied. We can of course also obtain numerical results for $f_{14}$ by solving the canonical system with DiffExp (as described in the previous section), but it would of course be interesting to obtain analytic results also for $f_{14}$.

We observe that the structure of the square root matches precisely the criteria of section 3.2. Indeed, the polynomial under the square root in eq. (5.1) has degree four in both $x$ and $y$. So, if we keep one variable fixed, the square root defines an elliptic curve in the other. This reflects the fact that the K3 surface defined by the square root is elliptically fibered [39]. As a consequence, we can choose the integration path in eq. (3.5) and solve the differential equation in terms of eMPLs. Before we do this, we have to make an important comment. While the strategy of section 3.1 to solve eq. (5.1) does not apply, it does not exclude that a solution in terms of MPLs evaluated at algebraic arguments exists. On the contrary, we have found that it is possible to evaluate $f_{14}$ from its Feynman parameter representation using direct integration techniques (cf., e.g., refs. [64-70]). ${ }^{4}$ The resulting expression, however, is extremely lengthy and involves MPLs evaluated at complicated algebraic arguments. We have not been able to confirm the final expression numerically, as its size and the complexity of the branch cuts renders the evaluation of the MPLs extremely challenging. Our representation obtained from direct integration is therefore not useful for practical purposes. As we will show now, the representation in terms of eMPLs is very compact.

Let us now explain how we can solve the system of differential equations for $\bar{f}$ in terms of MPLs and eMPLs. We first of all introduce new variables $(\bar{x}, \bar{y})=(1-x, 1-y)$, and use PolyLogTools [70] to express all MPLs of the form $G(\ldots ; x)$ or $G(\ldots ; y)$ in eq. (5.1) in terms of $G(\ldots ; \bar{x})$ and $G(\ldots ; \bar{y})$ respectively. For example, we find:

$$
G_{-y, 0,0}(x)=G_{2-\bar{y}, 1,1}(\bar{x})-\frac{\pi^{2}}{12} G_{1}(\bar{y})-G_{1,1,1}(\bar{y})+G_{1,1,2}(\bar{y})+\log 2 G_{1,1}(\bar{y})+\frac{3}{4} \zeta_{3} .
$$

We know from eq. (4.2) that $f_{14}$ must vanish for $s=t=0$, and so $\bar{f}(\bar{x}=0, \bar{y}=0)=0$. Our strategy is then as follows. We first use the differential equation in $\bar{y}$ to evolve $\bar{f}$ from $(\bar{x}, \bar{y})=(0,0)$ to $(\bar{x}, \bar{y})=\left(0, \bar{y}_{0}\right)$. We then use the differential equation in $\bar{x}$ to evolve from $(\bar{x}, \bar{y})=\left(0, \bar{y}_{0}\right)$ to the generic point $(\bar{x}, \bar{y})=\left(\bar{x}_{0}, \bar{y}_{0}\right)$. In the following we assume $0<\bar{x}_{0}, \bar{y}_{0}<1$ for concreteness (which corresponds to he Euclidean region $s, t<0$ ). On the line $\bar{x}=0$ we find

$$
\Delta(x=1, y)=\left(1-y^{2}\right)^{2}=\bar{y}^{2}(2-\bar{y})^{2} .
$$

\footnotetext{
${ }^{4}$ We are grateful to Erik Panzer for providing us with a change of variables that renders the Feynman parameter integral linearly reducible.
} 
Hence, the square root disappears in the limit $\bar{x} \rightarrow 0$, and we can solve the differential equation on the line $\bar{x}$ in terms of MPLs. We find (relabelling $\bar{y}_{0}$ as $\bar{y}$ ):

$$
\begin{aligned}
\bar{f}(\bar{x}=0, \bar{y})= & \left(2 \pi^{2} \log 2-3 \zeta_{3}\right) G_{1}(\bar{y})-\pi^{2} G_{1,1}(\bar{y})+2 \pi^{2} G_{1,2}(\bar{y}) \\
& +4 G_{1,0,1,1}(\bar{y})-4 G_{1,1,1,1}(\bar{y})+4 G_{1,2,1,1}(\bar{y}) .
\end{aligned}
$$

Next, let us discuss the solution on the line $\bar{y}=\bar{y}_{0}$. Clearly, $\Delta(x, y)$ is not a perfect square for $x \neq 0$. Looking at the constraint $\xi^{2}=\Delta\left(x, y_{0}\right)$ as a function of $x$ with $y_{0}$ fixed, we see that it defines an elliptic curve. The branch points, i.e., the zeroes in $\bar{x}$ of $\Delta\left(1-\bar{x}, 1-\bar{y}_{0}\right)$, are

$$
\vec{a}\left(\bar{y}_{0}\right)=\left(2-\bar{y}_{0}, \frac{\bar{y}_{0}\left(\bar{y}_{0}-\sqrt{\bar{y}_{0}^{2}+4 \bar{y}_{0}-4}\right)}{2\left(1-\bar{y}_{0}\right)}, \frac{\bar{y}_{0}\left(\bar{y}_{0}+\sqrt{\bar{y}_{0}^{2}+4 \bar{y}_{0}-4}\right)}{2\left(1-\bar{y}_{0}\right)}, \frac{2-\bar{y}_{0}}{1-\bar{y}_{0}}\right) .
$$

We can use eq. (3.16) to interpret every MPL of the form $G(\vec{b}(\bar{y}) ; \bar{x})$ as an eMPL of the form $\mathcal{E}_{4}(\underset{\vec{c}(\vec{y})}{\vec{n}} ; \bar{x}, \vec{a}(\bar{y}))$. Moreover, we can express all the algebraic coefficients multiplying the MPLs in the differential equation in terms of the functions $\Psi_{ \pm 1}(c, \bar{x}, \vec{a}(\bar{y}))$. For example, we find

$$
\begin{aligned}
\Psi_{-1}(\infty, \bar{x}, \vec{a}(\bar{y})) & =\frac{\bar{x}}{\bar{\xi}}-\frac{(y+1)^{2}}{4 y \xi} \\
\Psi_{-1}(0, \bar{x}, \vec{a}(\bar{y})) & =\frac{y^{2}-1}{\bar{x} y \xi}-\frac{y^{2}-1}{2 y \xi} \\
\Psi_{-1}(1, \bar{x}, \vec{a}(\bar{y})) & =\frac{(y-1)^{2}}{4 y \xi}-\frac{1}{(\bar{x}-1) \xi}
\end{aligned}
$$

with $\xi=\sqrt{\Delta(1-\bar{x}, 1-\bar{y})}$. In the process, we discover the relations:

$$
\begin{aligned}
Z_{4}(0, \vec{a}(\bar{y})) & =\frac{(2-\bar{y}) \bar{y}}{2 c_{4}(1-\bar{y})}=\frac{1-y^{2}}{2 y c_{4}}, \\
Z_{4}(1, \vec{a}(\bar{y})) & =\frac{\bar{y}^{2}}{4 c_{4}(1-\bar{y})}=\frac{(1-y)^{2}}{4 y c_{4}}, \\
G_{*}(\vec{a}(\bar{y})) & =\frac{(2-\bar{y})(3 \bar{y}-2)}{8 c_{4}(1-\bar{y})}=\frac{(1-y)(1-3 y)}{8 y c_{4}} .
\end{aligned}
$$

At this point we need to make an important comment about how we choose the branches of the square root $\xi=\sqrt{1-\bar{x}, 1-\bar{y})}$. From eq. (5.6) we can see that for $0<y<3-2 \sqrt{2}$, the four branch points are real and ordered according to $a_{1}(y)<a_{2}(y)<a_{3}(y)<a_{4}(y)$. The branches of the square root are chosen according to

$$
\xi=\sqrt{\Delta(x, y)}=\sqrt{|\Delta(x, y)|} \times\left\{\begin{array}{c}
-1, x<a_{1}(y) \text { or } x \geq a_{4}(y) \\
-i, a_{1}(y) \leq x<a_{2}(y) \\
1, a_{2}(y) \leq x<a_{3}(y) \\
i, a_{3}(y) \leq x<a_{4}(y)
\end{array}\right.
$$


For $3-2 \sqrt{3}<y<1$, we have $a_{1}\left(\bar{y}_{0}\right), a_{4}\left(\bar{y}_{0}\right)>0$ and $a_{2}\left(\bar{y}_{0}\right)^{*}=a_{3}\left(\bar{y}_{0}\right)$, with $\operatorname{Im} a_{3}\left(\bar{y}_{0}\right)>0$. The branches of the square are then chosen as:

$$
\xi=\sqrt{\Delta(x, y)}=\sqrt{|\Delta(x, y)|} \times\left\{\begin{array}{l}
-1, x<a_{1}(y) \text { or } x \geq a_{4}(y) \\
-i, a_{1}(y) \leq x<a_{4}(y)
\end{array}\right.
$$

We stress that part of this choice is purely conventional, and compensated by the leading singularity $1 / \sqrt{\Delta(x, y)}$ when passing from the master integral $g_{14}$ to its canonical analogue $f_{14}$.

The resulting differential equation can be solved by quadrature, and all integrals over $\bar{x}$ can be performed in terms of eMPLs using eq. (3.11). The final result reads (we relabel again $\left(x_{0}, y_{0}\right)$ as $\left.(x, y)\right)$ :

$$
\begin{aligned}
& \bar{f}(x, y)=2 \mathcal{E}_{4}\left(\begin{array}{cccc}
-1 & 1 & 1 & 1 \\
\infty & \frac{1}{y}+1 & 1 & 1
\end{array} ; \bar{x}, \vec{a}\right)+2 \mathcal{E}_{4}\left(\begin{array}{cccc}
-1 & 1 & 1 & 1 \\
\infty & y+1 & 1 & 1
\end{array} ; \bar{x}, \vec{a}\right)+2 \mathcal{E}_{4}\left(\begin{array}{cccc}
-1 & 1 & 1 & 1 \\
1 & \frac{1}{y}+1 & 1 & 1
\end{array} ; \bar{x}, \vec{a}\right) \\
& +2 \mathcal{E}_{4}\left(\begin{array}{cccc}
-1 & 1 & 1 & 1 \\
1 & y+1 & 1 & 1
\end{array} ; \bar{x}, \vec{a}\right)+4 \mathcal{E}_{4}\left(\begin{array}{cccc}
-1 & 1 & 1 & 1 \\
\infty & 0 & 1 & 1
\end{array} ; \bar{x}, \vec{a}\right)-4 \mathcal{E}_{4}\left(\begin{array}{cccc}
-1 & 1 & 1 & 1 \\
\infty & 1 & 1 & 1
\end{array} ; \bar{x}, \vec{a}\right) \\
& +4 \mathcal{E}_{4}\left(\begin{array}{cccc}
-1 & 1 & 1 & 1 \\
1 & 0 & 1 & 1
\end{array} ; \bar{x}, \vec{a}\right)-4 \mathcal{E}_{4}\left(\begin{array}{cccc}
-1 & 1 & 1 & 1 \\
1 & 1 & 1 & 1
\end{array} \bar{x}, \vec{a}\right)-\left(3 \log ^{2} y+\pi^{2}\right) \mathcal{E}_{4}\left(\begin{array}{cc}
-1 & 1 \\
\infty & 1
\end{array} ; \bar{x}, \vec{a}\right) \\
& +\left(\log ^{2} y+\pi^{2}\right)\left[\mathcal{E}_{4}\left(\begin{array}{cc}
-1 & 1 \\
\infty & \frac{1}{y}+1
\end{array} ; \bar{x}, \vec{a}\right)+\mathcal{E}_{4}\left(\begin{array}{cc}
-1 & 1 \\
\infty & y+1
\end{array} ; \bar{x}, \vec{a}\right)+\mathcal{E}_{4}\left(\begin{array}{cc}
-1 & 1 \\
1 & \frac{1}{y}+1
\end{array} ; \bar{x}, \vec{a}\right)\right. \\
& \left.+\mathcal{E}_{4}\left(\begin{array}{cc}
-1 & 1 \\
1 & y+1
\end{array} ; \bar{x}, \vec{a}\right)\right]+\left(\log ^{2} y-\pi^{2}\right) \mathcal{E}_{4}\left(\begin{array}{cc}
-1 & 1 \\
1 & 1
\end{array} \bar{x}, \vec{a}\right)+2 \log y\left[\mathcal{E}_{4}\left(\begin{array}{ccc}
-1 & 1 & 1 \\
\infty & \frac{1}{y}+1 & 1
\end{array} ; \bar{x}, \vec{a}\right)\right. \\
& \left.-\mathcal{E}_{4}\left(\begin{array}{ccc}
-1 & 1 & 1 \\
\infty & y+1 & 1
\end{array} ; \bar{x}, \vec{a}\right)+2 \mathcal{E}_{4}\left(\begin{array}{ccc}
-1 & 1 & 1 \\
0 & 1 & 1
\end{array} ; \bar{x}, \vec{a}\right)+\mathcal{E}_{4}\left(\begin{array}{ccc}
-1 & 1 & 1 \\
1 & \frac{1}{y}+1 & 1
\end{array} ; \bar{x}, \vec{a}\right)-\mathcal{E}_{4}\left(\begin{array}{ccc}
-1 & 1 & 1 \\
1 & y+1 & 1
\end{array} ; \bar{x}, \vec{a}\right)\right] \\
& +\left[-4 \operatorname{Li}_{3}(-y)-4 \operatorname{Li}_{3}(y)+4 \operatorname{Li}_{2}(-y) \log y+4 \operatorname{Li}_{2}(y) \log y-\frac{2}{3} \log ^{3} y\right. \\
& +2 \log (1-y) \log ^{2} y+2 \log (y+1) \log ^{2} y-\pi^{2} \log y+2 \pi^{2} \log (y+1) \\
& \left.-2 \zeta_{3}\right]\left[\mathcal{E}_{4}(\underset{\infty}{-1} ; \bar{x}, \vec{a})+\mathcal{E}_{4}(\underset{1}{-1} ; \bar{x}, \vec{a})\right] \\
& -12 \mathrm{Li}_{4}(-y)-12 \mathrm{Li}_{4}(y)-2 \mathrm{Li}_{2}(y) \log ^{2} y-2 \operatorname{Li}_{2}(-y)\left(\log ^{2} y+\pi^{2}\right) \\
& +8 \mathrm{Li}_{3}(-y) \log y+8 \mathrm{Li}_{3}(y) \log y-2 \zeta_{3} \log y-\frac{1}{6} \log ^{4} y-\frac{1}{2} \pi^{2} \log ^{2} y-\frac{3 \pi^{4}}{20},
\end{aligned}
$$

where we introduced the shorthand $\vec{a} \equiv \vec{a}(\bar{y})$, and we have replaced all MPLs by classical polylogarithms:

$$
\operatorname{Li}_{n}(z)=-G(\underbrace{0, \ldots, 0}_{n-1}, 1 ; z) .
$$

We have checked numerically that our final result for $\bar{f}(x, y)$ is correct by comparing against Fiesta. The eMPLs in eq. (5.11) were evaluated numerically using an in-house code (a public numerical implementation of eMPLs into GiNaC exists [71]). Note that $\bar{f}(x, y)$ is a pure function of uniform weight four [49]. We find it interesting that such a compact analytic expression in terms of eMPLs exists, while our MPL expression obtained by naive direct integration is prohibitively large.

Let us comment on the usefulness of our compact analytic result in terms of eMPLs. eMPLs have been introduced into the physics literature only very recently. For this reason, their algebraic properties and their efficient numerical evaluation are much less understood 


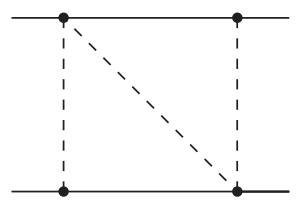

Figure 3. The graph associated with the integral $B\left(s, t, m^{2}\right)$ in eq. (5.13).

than in the case of MPLs. It may thus seem that having an analytic expression in terms of eMPLs is of no use. We argue that this is not so: eMPLs are currently an active topic of research in both mathematics and physics. For example, first proposals have been made how some of the mathematical tools used to manipulate MPLs, like the coaction [19, 72 74] and the symbol [18, 36, 64, 75-77], can be extended to eMPLs [27]. Moreover, first public numeric implementation of eMPLs have become available [71]. We foresee that the structure of eMPLs will be much better understood in the future, and it may become possible to manipulate and evaluate eMPLs with the same ease as MPLs. In this scenario, our compact analytic expression will unfold its full power and become superior to a lengthy and clumsy expression in terms or ordinary MPLs.

\subsection{A compact analytic result for the first planar family}

Let us conclude this paper by applying the techniques from section 5.1 to the planar family in figure 1(a). In ref. [16] it was shown that all integrals in this family can be expressed in terms of 23 master integrals, and all but one master integral were evaluated in terms of MPLs. The remaining master integral that could not be expressed in terms of MPLs in ref. [16] is (see figure 3):

$$
B\left(s, t, m^{2}\right)=\frac{e^{2 \gamma_{E} \epsilon}}{\pi^{D}} \int \frac{d^{D} k_{1} d^{D} k_{2}}{\left[\left(k_{1}+p_{1}+p_{2}\right)^{2}-m^{2}\right]\left[k_{2}^{2}-m^{2}\right]\left(k_{1}+p_{1}\right)^{2}\left(k_{1}-k_{2}\right)^{2}\left(k_{2}-p_{3}\right)^{2}} .
$$

This integral was evaluated in terms of a rather lengthy combination of MPLs with algebraic arguments in ref. [24]. Here we show that, by using the same strategy as in section 5.1, we can obtain a very compact representation for $B\left(s, t, m^{2}\right)$ in terms of the same type of eMPLs as in eq. (5.11).

The integral in eq. (5.13) is finite in four dimensions. Let us define $\widetilde{B}(x, y)$ by

$$
B\left(s, t, m^{2}\right)=\frac{1}{4 \sqrt{(s+t)\left(s+t-4 m^{2}\right)}} \widetilde{B}(x, y)+\mathcal{O}(\epsilon)
$$

where the Landau variables $(x, y)$ have been defined in eq. (4.1). Our starting point is the differential equation satisfied by $\widetilde{B}(x, y)[16]$ :

$$
\begin{aligned}
d \widetilde{B}(x, y)= & g_{1} d \log \frac{1-Q(x, y)}{1+Q(x, y)}+g_{2} d \log \frac{(1+x)+(1-x) Q(x, y)}{(1+x)-(1-x) Q(x, y)} \\
& +g_{3} d \log \frac{(1+y)+(1-y) Q(x, y)}{(1+y)-(1-y) Q(x, y)}
\end{aligned}
$$


The functions $g_{i}$ appearing in the right-hand side of eq. (5.15) are pure combinations of MPLs of weight three,

$$
\begin{aligned}
g_{1}= & -16 G_{0}(y) G_{0,0}(x)+32 G_{1}(y) G_{0,0}(x)+8 G_{0,0,0}(y)+16 G_{0,0,1}(y) \\
& -32 G_{0,-1,0}(x)+8 G_{0,0,0}(x)+16 G_{1,0,0}(x)+\frac{8}{3} \pi^{2} G_{0}(y)-\frac{16}{3} \pi^{2} G_{1}(y) \\
& -\frac{8}{3} \pi^{2} G_{0}(x)-32 \zeta_{3}, \\
g_{2}= & -16 G_{0,0,0}(x)-\frac{8}{3} \pi^{2} G_{0}(x), \\
g_{3}= & 8 G_{0}(x) G_{-\frac{1}{x}, 0}(y)+16 G_{0}(x) G_{-\frac{1}{x}, 1}(y)-8 G_{0}(x) G_{-x, 0}(y)-\frac{4}{3} \pi^{2} G_{0}(x) \\
& -16 G_{0}(x) G_{-x, 1}(y)+16 G_{-1,0}(x) G_{-\frac{1}{x}}(y)-16 G_{-1,0}(x) G_{-x}(y)-24 \zeta_{3} \\
& -8 G_{0}(y) G_{0,0}(x)-8 G_{0,0}(x) G_{-\frac{1}{x}}(y)+8 G_{0,0}(x) G_{-x}(y)+8 G_{-\frac{1}{x}, 0,0}(y) \\
& +16 G_{-\frac{1}{x}, 0,1}(y)+8 G_{-x, 0,0}(y)+16 G_{-x, 0,1}(y)-16 G_{-1,0,0}(y)-32 G_{-1,0,1}(y) \\
& -16 G_{0,-1,0}(x)+8 G_{0,0,0}(x)+\frac{20}{3} \pi^{2} G_{-\frac{1}{x}}(y)+4 \pi^{2} G_{-x}(y)-\frac{32}{3} \pi^{2} G_{-1}(y) .
\end{aligned}
$$

The function $Q(x, y)$ is related to exactly the same square root defined in eqs. (5.1) and (5.2):

$$
Q(x, y)=\sqrt{\frac{(x+y)(1+x y)}{x^{2} y+x y^{2}-4 x y+x+y}}=\frac{\sqrt{\Delta(x, y)}}{x^{2} y+x y^{2}-4 x y+x+y},
$$

and the initial condition is $\widetilde{B}(x=1, y=1)=0$. Following exactly the same steps as in section 5.1, we find the following very compact expression:

$$
\begin{aligned}
& \widetilde{B}(x, y)=16 \log \frac{-t}{m^{2}}\left[\mathcal{E}_{4}\left(\begin{array}{ccc}
-1 & 1 & 1 \\
0 & 1+1 / y & 1
\end{array} ; \bar{x}, \vec{a}\right)-\mathcal{E}_{4}\left(\begin{array}{rrr}
-1 & 1 & 1 \\
0 & 1+y & 1
\end{array} ; \bar{x}, \vec{a}\right)\right. \\
& \left.+\mathcal{E}_{4}\left(\begin{array}{ccc}
-1 & 1 & 1 \\
\infty & 1 & 1
\end{array} ; \bar{x}, \vec{a}\right)+\mathcal{E}_{4}\left(\begin{array}{ccc}
-1 & 1 & 1 \\
1 & 1 & 1
\end{array} ; \bar{x}, \vec{a}\right)+\zeta_{2} \mathcal{E}_{4}\left(-{ }_{\infty}^{-1} ; \bar{x}, \vec{a}\right)+\zeta_{2} \mathcal{E}_{4}\left(\begin{array}{c}
-1 \\
1
\end{array} ; \bar{x}, \vec{a}\right)\right] \\
& -8\left(8 \zeta_{2}+4 \operatorname{Li}_{2}(y)+\log ^{2} y\right)\left[\mathcal{E}_{4}\left(\begin{array}{cc}
-1 & 1 \\
0 & 1+1 / y
\end{array} ; \bar{x}, \vec{a}\right)+\mathcal{E}_{4}\left(\begin{array}{cc}
-1 & 1 \\
0 & 1+y
\end{array} ; \bar{x}, \vec{a}\right)-\mathcal{E}_{4}\left(\begin{array}{cc}
-1 & 1 \\
0 & 1
\end{array} ; \bar{x}, \vec{a}\right)\right] \\
& -32 \zeta_{2}\left[\mathcal{E}_{4}\left(\begin{array}{cc}
-1 & 1 \\
\infty & 1
\end{array} ; \bar{x}, \vec{a}\right)-\mathcal{E}_{4}\left(\begin{array}{cc}
-1 & 1 \\
1 & 1
\end{array} ; \bar{x}, \vec{a}\right)\right]+16 \mathcal{E}_{4}\left(\begin{array}{cccc}
-1 & 1 & 1 & 1 \\
0 & 1+1 / y & 1 & 1
\end{array} ; \bar{x}, \vec{a}\right) \\
& -32 \mathcal{E}_{4}\left(\begin{array}{ccccc}
-1 & 1 & 1 & 1 \\
0 & 1+1 / y & 2 & 1
\end{array} ; \bar{x}, \vec{a}\right)-16 \mathcal{E}_{4}\left(\begin{array}{cccc}
-1 & 1 & 1 & 1 \\
0 & 1+y & 1 & 1
\end{array} ; \bar{x}, \vec{a}\right)+32 \mathcal{E}_{4}\left(\begin{array}{cccc}
-1 & 1 & 1 & 1 \\
0 & 1+y & 2 & 1
\end{array} ; \vec{x}, \vec{a}\right) \\
& +16 \mathcal{E}_{4}\left(\begin{array}{cccc}
-1 & 1 & 1 & 1 \\
\infty & 0 & 1 & 1
\end{array} ; \bar{x}, \vec{a}\right)-24 \mathcal{E}_{4}\left(\begin{array}{cccc}
-1 & 1 & 1 & 1 \\
\infty & 1 & 1 & 1
\end{array} ; \bar{x}, \vec{a}\right)-32 \mathcal{E}_{4}\left(\begin{array}{cccc}
-1 & 1 & 1 & 1 \\
\infty & 1 & 2 & 1
\end{array} ; \bar{x}, \vec{a}\right) \\
& +16 \mathcal{E}_{4}\left(\begin{array}{ccccc}
-1 & 1 & 1 & 1 \\
1 & 0 & 1 & 1
\end{array} ; \bar{x}, \vec{a}\right)+40 \mathcal{E}_{4}\left(\begin{array}{ccccc}
-1 & 1 & 1 & 1 \\
1 & 1 & 1 & 1
\end{array} ; \bar{x}, \vec{a}\right)-32 \mathcal{E}_{4}\left(\begin{array}{cccc}
-1 & 1 & 1 & 1 \\
1 & 1 & 2 & 1
\end{array} ; \bar{x}, \vec{a}\right) \\
& +\frac{4}{3}\left(12 \operatorname{Li}_{3}(y)+24 \zeta_{2} \log y+\log ^{3} y\right)\left[\mathcal{E}_{4}(\underset{\infty}{-1} ; \bar{x}, \vec{a})+\mathcal{E}_{4}(\underset{1}{-1} ; \bar{x}, \vec{a})\right] \\
& +64 \zeta_{4}-32 \zeta_{2} \operatorname{Li}_{2}(y)+16 \operatorname{Li}_{4}(y)+8 \zeta_{2} \log ^{2} y+\frac{1}{3} \log ^{4} y .
\end{aligned}
$$

We have again checked our result numerically. Note that again we find a pure function of uniform weight four. 


\section{Conclusions}

In this paper we considered the computation of the second family of planar master integrals relevant for Bhabha scattering at two loops in QED including the full dependence on the electron mass. Our primary tool for their analytic study was the method of differential equations, augmented by the choice of a canonical basis. We described how we obtained our canonical basis and showed that four different square roots appear in the calculation of the 43 master integrals that comprise it. We also provided boundary conditions at $s=t=0$ for all master integrals. Together with the matrices defining the differential equations, this input is sufficient to produce high-precision numerical results for all master integrals and for all kinematic regions using the public code DiffExp. We provide a Mathematica notebook that allows one to evaluate all master integrals numerically with DiffExp as supplementary material attached to this paper.

We also considered the analytic solution of the differential equations. Interestingly, the four square roots never appear all at the same time, and the differential equations for all master integrals but one can be solved in terms of MPLs by rationalising three of the four square roots by suitable changes of variables. For the contributions up to weight three, this procedure leads to analytic results which we present as supplementary material attached to this paper. While conceptually straightforward, we find that this procedure generates rather involved analytical expressions for the weight four part of the master integrals. The analytic results are available in electronic form from the authors upon request.

In the last part of the paper, we focused on the analytic computation of the remaining master integral, whose canonical differential equations contain three different square roots which cannot be rationalised at the same time. An analytic calculation in terms of MPLs cannot be easily obtained by direct integration of the differential equations due to the non-rationalisable square root. Instead, we show that compact analytic expressions can be obtained algorithmically in terms of elliptic multiple polylogarithms. We applied this idea in detail to our problem and obtained in this way a very compact analytic expression for this integral. We also showed that a similar compact expression can be obtained for another planar integral relevant for two-loop Bhabha scattering, whose calculation in terms of (a lengthy combination of) MPLs had been considered some years ago.

This paper concludes the analytic calculation of the planar master integrals for Bhabha scattering at two loops. For the future, it would be interesting to complete also the computation of the non-planar two-loop family. Once this last step is achieved, we will have all the ingredients to obtain for the first time complete two-loop results in QED for one of the standard candle processes at an electron-positron collider.

\section{Acknowledgments}

We are grateful to Martijn Hidding for help in applying DiffExp and to Erik Panzer for suggesting the change of variable that renders the integral $f_{14}$ linearly reducible. We thank Marco Besier, Jake Bourjaily, Johannes Brödel, Falko Dulat and Brenda Penante for discussions. The work of V.S. was supported by the Russian Science Foundation, agreement 
no. 21-71-30003 (checking results with updated version of FIESTA) and by the Ministry of Education and Science of the Russian Federation as part of the program of the Moscow Center for Fundamental and Applied Mathematics under agreement no. 075-15-2019-1621 (solving differential equations).

\section{A Canonical basis}

In this appendix we show our choice of canonical basis. Note that we prefer to choose the square roots in such a form that they are manifestly real in the Euclidean region $s, t<0$. This holds also for the boundary conditions which we presented in eq. (4.2).

$$
\begin{aligned}
& f_{1}=\epsilon^{2} F_{2,0,0,0,0,2,0,0,0}, \\
& f_{2}=-\epsilon^{2} \frac{1}{2} \sqrt{-s} \sqrt{4 m^{2}-s} F_{0,2,1,0,0,2,0,0,0}-\epsilon^{2} \sqrt{-s} \sqrt{4 m^{2}-s} F_{0,2,2,0,0,1,0,0,0}, \\
& f_{3}=-\epsilon^{2} s F_{0,2,1,0,0,2,0,0,0} \text {, } \\
& f_{4}=-\frac{1}{2} \epsilon^{2} \sqrt{-t} \sqrt{4 m^{2}-t} F_{0,0,0,0,1,2,2,0,0}-\epsilon^{2} \sqrt{-t} \sqrt{4 m^{2}-t} F_{0,0,0,0,2,1,2,0,0}, \\
& f_{5}=-\epsilon^{2} t F_{0,0,0,0,1,2,2,0,0} \\
& f_{6}=-\epsilon^{2} m^{2} F_{0,0,1,0,2,2,0,0,0} \\
& f_{7}=-\epsilon^{3} \sqrt{-s} \sqrt{4 m^{2}-s} F_{0,1,1,0,1,2,0,0,0}, \\
& f_{8}=-\epsilon^{3} \sqrt{-t} \sqrt{4 m^{2}-t} F_{0,0,1,0,1,2,1,0,0}, \\
& f_{9}=-\epsilon^{2} m^{2} F_{2,0,0,0,0,2,1,0,0}, \\
& f_{10}=-\epsilon^{2} m^{2} F_{2,0,0,0,0,2,1,0,0} \text {, } \\
& f_{11}=-\epsilon^{3} \sqrt{-s} \sqrt{4 m^{2}-s} F_{0,1,1,0,0,2,1,0,0}, \\
& f_{12}=2 \epsilon^{3} t F_{0,1,0,0,1,1,2,0,0}-\epsilon^{2} t F_{0,1,0,-1,1,2,2,0,0} \text {, } \\
& f_{13}=-\epsilon^{3} \sqrt{-t} \sqrt{4 m^{2}-t} F_{0,1,0,0,1,1,2,0,0}, \\
& f_{14}=-\epsilon^{4} \sqrt{-s-t} \sqrt{4 m^{2}-s-t} F_{0,1,1,0,1,1,1,0,0}, \\
& f_{15}=\frac{1}{2} \epsilon^{3} \sqrt{-t} \sqrt{4 m^{2}-t}\left(2 m^{2} F_{0,1,1,0,1,2,1,0,0}+2 m^{2} F_{0,2,1,0,1,1,1,0,0}-s F_{0,1,1,0,1,2,1,0,0}\right) \text {, } \\
& f_{16}=\epsilon^{3} \sqrt{-s} \sqrt{-t} \sqrt{4 m^{2}-s} \sqrt{4 m^{2}-t} F_{0,1,1,0,1,2,1,0,0}, \\
& f_{17}=\frac{1}{2} \epsilon^{3} \sqrt{-s} \sqrt{4 m^{2}-s}\left(2 m^{2} F_{0,1,1,0,1,1,2,0,0}+2 m^{2} F_{0,1,1,0,1,2,1,0,0}-t F_{0,1,1,0,1,2,1,0,0}\right), \\
& f_{18}=\frac{\epsilon^{3} m^{2}\left(4 m^{2}-t\right)}{2\left(4 m^{2}-s-t\right)} F_{0,0,1,0,1,2,1,0,0}-\frac{\epsilon^{3}\left(4 m^{2}-t\right)\left(2 m^{2}-s-t\right)}{2\left(4 m^{2}-s-t\right)} F_{0,1,0,0,1,1,2,0,0} \\
& +\frac{\epsilon^{3} m^{2} s\left(4 m^{2}-s\right)}{t\left(4 m^{2}-s-t\right)} F_{0,1,1,0,0,2,1,0,0}-\epsilon^{3}\left(\epsilon 10 m^{2}-m^{2}-3 \epsilon s-3 \epsilon t\right) F_{0,1,1,0,1,1,1,0,0} \\
& -\frac{\epsilon^{3} m^{2}(s+t)}{t} F_{0,2,1,0,1,1,1,0,-1}+\frac{\epsilon^{2} m^{2}\left(4 m^{2}-t\right)}{4\left(4 m^{2}-s-t\right)} F_{0,0,0,0,1,2,2,0,0} \\
& +\frac{\epsilon^{3} m^{2}\left(8 m^{4}-4 m^{2} s-6 m^{2} t+s^{2}+2 s t+t^{2}\right)}{4 m^{2}-s-t} F_{0,2,1,0,1,1,1,0,0}
\end{aligned}
$$




$$
\begin{aligned}
& +\frac{\epsilon^{2} m^{2}\left(4 m^{2}-t\right)}{2\left(4 m^{2}-s-t\right)} F_{0,0,0,0,2,1,2,0,0}+\frac{\epsilon^{3} m^{2}\left(8 m^{4}-4 m^{2} s-2 m^{2} t+s^{2}+s t\right)}{4 m^{2}-s-t} F_{0,1,1,0,1,1,2,0,0} \\
& +\frac{\epsilon^{3}\left(8 m^{4}-4 m^{2} s-2 m^{2} t+s^{2}+s t\right)}{4\left(4 m^{2}-s-t\right)} F_{0,1,1,0,1,2,0,0,0} \\
& +\frac{\epsilon^{2}\left(8 m^{4}-4 m^{2} s-2 m^{2} t+s^{2}+s t\right)}{8\left(4 m^{2}-s-t\right)} F_{0,2,1,0,0,2,0,0,0} \\
& +\frac{\epsilon^{2}\left(8 m^{4}-4 m^{2} s-2 m^{2} t+s^{2}+s t\right)}{4\left(4 m^{2}-s-t\right)} F_{0,2,2,0,0,1,0,0,0} \\
& +\frac{1}{2} \epsilon^{3}\left(8 m^{4}-2 m^{2} s-2 m^{2} t+s t\right) F_{0,1,1,0,1,2,1,0,0}
\end{aligned}
$$

$f_{19}=-\epsilon^{2} s F_{2,0,2,1,0,0,0,0,0}$,

$f_{20}=\epsilon^{2} \sqrt{-s} s \sqrt{4 m^{2}-s} F_{2,1,2,1,0,0,0,0,0}$,

$f_{21}=-\epsilon^{2} \sqrt{-s} \sqrt{4 m^{2}-s} F_{2,1,0,0,0,2,0,0,0}$,

$f_{22}=-\frac{1}{2} \epsilon^{3} s F_{0,0,1,1,2,1,0,0,0}-\epsilon^{2} s F_{0,0,2,1,1,2,0,-1,0}$,

$f_{23}=-\epsilon^{3} \sqrt{-s} \sqrt{4 m^{2}-s} F_{0,0,1,1,2,1,0,0,0}$,

$f_{24}=-\epsilon^{3} \sqrt{-s} \sqrt{4 m^{2}-s} F_{0,0,1,1,1,2,0,0,0}$,

$f_{25}=-\epsilon^{3} \sqrt{-s} \sqrt{4 m^{2}-s} F_{2,0,1,1,0,0,1,0,0}$,

$f_{26}=\epsilon^{3} s F_{1,1,0,0,0,2,1,0,0}-\epsilon^{2} s F_{2,1,0,0,0,2,1,0,-1}$,

$f_{27}=-\epsilon^{3} \sqrt{-s} \sqrt{4 m^{2}-s} F_{1,1,0,0,0,2,1,0,0}$,

$f_{28}=\epsilon^{3} s F_{1,1,1,1,0,1,0,0,0}-2 s \epsilon^{4} F_{1,1,1,1,0,1,0,0,0}$,

$f_{29}=-\epsilon^{4} \sqrt{-s} \sqrt{4 m^{2}-s} F_{1,0,1,1,1,1,0,0,0}$,

$f_{30}=\epsilon^{4} s^{2} F_{1,1,1,1,1,1,0,0,0}-4 \epsilon^{4} m^{2} s F_{1,1,1,1,1,1,0,0,0}$,

$f_{31}=\epsilon^{3} s^{2} F_{2,1,1,1,0,0,1,0,0}-4 \epsilon^{3} m^{2} s F_{2,1,1,1,0,0,1,0,0}$,

$f_{32}=\epsilon^{4} \sqrt{-s} s \sqrt{4 m^{2}-s} F_{1,1,1,1,0,1,1,0,0}$

$f_{33}=-\epsilon^{3} \sqrt{-s} \sqrt{4 m^{2}-s} F_{1,1,0,0,1,2,1,0,-1}-\epsilon^{3} \sqrt{-s} t \sqrt{4 m^{2}-s} F_{1,1,0,0,1,2,1,0,0}$,

$f_{34}=\epsilon^{3} \sqrt{-s} \sqrt{-t} \sqrt{4 m^{2}-s} \sqrt{4 m^{2}-t} F_{1,1,0,0,1,2,1,0,0}$,

$f_{35}=-\epsilon^{3} \sqrt{-s} \sqrt{4 m^{2}-s} F_{0,0,1,1,1,2,1,-1,0}$,

$f_{36}=\epsilon^{3} s \sqrt{-t} \sqrt{4 m^{2}-t} F_{0,0,1,1,1,2,1,0,0}+\epsilon^{3} s \sqrt{-t} \sqrt{4 m^{2}-t} F_{0,0,1,1,2,1,1,0,0}$,

$f_{37}=\epsilon^{3} \sqrt{-s} \sqrt{4 m^{6}-s\left(m^{2}-t\right)^{2}} F_{0,0,1,1,1,2,1,0,0}$,

$f_{38}=\epsilon^{4} s \sqrt{-t} \sqrt{4 m^{2}-t} F_{1,0,1,1,1,1,1,0,0}$,

$f_{39}=-\epsilon^{4} \sqrt{-s} \sqrt{4 m^{2}-s}\left(m^{2} F_{1,0,1,1,1,1,1,0,0}-F_{0,1,1,0,1,1,1,0,0}+F_{1,0,1,1,1,1,1,-1,0}\right)$,

$f_{40}=-\epsilon^{4} s \sqrt{-s} \sqrt{-t} \sqrt{4 m^{2}-s} \sqrt{4 m^{2}-t} F_{1,1,1,1,1,1,1,0,0}$,

$f_{41}=-\epsilon^{4} s \sqrt{-s} \sqrt{4 m^{2}-s}\left(F_{1,1,1,1,1,1,1,0,-1}+t F_{1,1,1,1,1,1,1,0,0}\right)$,

$f_{42}=\epsilon^{4}\left(4 m^{4} s F_{1,1,1,1,1,1,1,0,0}-m^{2} s^{2} F_{1,1,1,1,1,1,1,0,0}\right.$

$\left.+4 m^{2} s F_{1,1,1,1,1,1,1,-1,0}-s^{2} F_{1,1,1,1,1,1,1,-1,0}\right)$, 


$$
\begin{aligned}
f_{43}= & \epsilon^{4} m^{2} s F_{1,0,1,1,1,1,1,0,0}-\epsilon^{4} m^{2} s F_{1,1,1,1,1,1,1,0,-1}-\epsilon^{3} m^{2} s F_{0,1,1,0,1,1,2,0,0} \\
& -\epsilon^{3} m^{2} s F_{0,1,1,0,1,2,1,0,0}+\epsilon^{4} \frac{1}{2} s^{2} t F_{1,1,1,1,1,1,1,0,0}-\epsilon^{4} \frac{1}{2} s^{2} F_{1,1,1,1,0,1,1,0,0} \\
& +\epsilon^{4} \frac{1}{2} s^{2} F_{1,1,1,1,1,1,1,0,-1}+\frac{1}{2} \epsilon^{3} s t F_{0,1,1,0,1,2,1,0,0}-\epsilon^{3} s t F_{1,1,0,0,1,2,1,0,0} \\
& +\epsilon^{3} s t F_{2,1,1,1,0,0,1,0,0}+\epsilon^{4} s F_{0,1,1,0,1,1,1,0,0},-\epsilon^{4} s F_{1,0,1,1,1,1,0,0,0}+\epsilon^{4} s F_{1,0,1,1,1,1,1,-1,0} \\
& -\epsilon^{4} s F_{1,1,1,1,1,1,1,-1,-1}-\frac{1}{2} \epsilon^{3} s F_{0,1,1,0,0,2,1,0,0}+\frac{1}{4} \epsilon^{3} s F_{0,1,1,0,1,2,0,0,0}+\epsilon^{3} s F_{1,1,0,0,0,2,1,0,0} \\
& -\epsilon^{3} s F_{1,1,0,0,1,2,1,0,-1}+\frac{1}{2} \epsilon^{3} s F_{1,1,1,1,0,1,0,0,0}-\epsilon^{2} \frac{1}{8} s F_{0,2,1,0,0,2,0,0,0}-\epsilon^{2} \frac{1}{4} s F_{0,2,2,0,0,1,0,0,0}
\end{aligned}
$$

\section{B Elliptic multiple polylogarithms}

In this appendix we collect some technical material related to eMPLs not reviewed in section 3.2 .

In the case where all the indices $A_{i}=\left(\begin{array}{c}n_{i} \\ c_{i}\end{array}\right)$ are equal to $\left(\begin{array}{c} \pm 1 \\ 0\end{array}\right)$, the integral in eq. (3.11) is divergent and requires a special treatment and we define instead,

$$
\begin{aligned}
\mathcal{E}_{4}\left(A_{1} \ldots A_{k} ; x ; \vec{a}\right)=\frac{1}{k !} \log ^{k} x+ & \sum_{l=0}^{k} \sum_{m=1}^{l} \sum_{\sigma} \frac{(-1)^{l+m}}{(k-l) !} \log ^{k-l} x \\
& \times \mathcal{E}_{4}^{\mathrm{R}}\left(A_{\sigma(1)}^{(m)} \ldots A_{\sigma(m-1)}^{(m)} A_{\sigma(m+1)}^{(m)} \ldots A_{\sigma(l)}^{(m)} \mid A_{m} ; x ; \vec{a}\right),
\end{aligned}
$$

with $A_{i}^{(m)}=A_{i}$ if $i<m$ and $A_{i}^{(m)}=\left(\begin{array}{l}1 \\ 0\end{array}\right)$ otherwise. The third sum runs over all shuffles $\sigma$ of $\left(A_{1}^{(m)} \ldots A_{m-1}^{(m)}\right)$ and $\left(A_{m+1}^{(m)} \ldots A_{l}^{(m)}\right)$, i.e., over all permutations of their union that preserve the relative ordering within each of the two lists. The $\mathcal{E}_{4}^{\mathrm{R}}$ are iterated integrals with suitable subtractions to render the integrations finite,

$$
\mathcal{E}_{4}^{\mathrm{R}}\left(\begin{array}{cccc}
n_{1} & \ldots & n_{k} \\
0 & \ldots & 0
\end{array} \mid \begin{array}{c}
n_{a} \\
0
\end{array} ; x ; \vec{a}\right)=\int_{0}^{x} d t_{1} \Psi_{n_{1}}\left(0, t_{1}\right) \int_{0}^{t_{1}} \ldots \int_{0}^{t_{k-1}} d t_{k}\left(\Psi_{n_{a}}\left(0, t_{k}\right)-\Psi_{1}\left(0, t_{k}\right)\right) .
$$

The rather ad-hoc looking form of eq. (B.1) is determined essentially uniquely by requiring the regularised eMPLs to share the same algebraic and differential properties as their convergent analogues. We refer to ref. [49] for a detailed discussion.

The functions $Z_{4}(x, \vec{a})$ and $G_{*}(\vec{a})$ that appear in eq. (3.15) can be expressed in terms of incomplete elliptic integrals of the first and second kind [25, 49]:

$$
\begin{aligned}
& \mathrm{F}(x \mid \lambda)=\int_{0}^{x} \frac{d t}{\sqrt{\left(1-t^{2}\right)\left(1-\lambda t^{2}\right)}} \\
& \mathrm{E}(x \mid \lambda)=\int_{0}^{x} d t \sqrt{\frac{1-\lambda t^{2}}{1-t^{2}}}
\end{aligned}
$$

Open Access. This article is distributed under the terms of the Creative Commons Attribution License (CC-BY 4.0), which permits any use, distribution and reproduction in any medium, provided the original author(s) and source are credited. 


\section{References}

[1] P. Banerjee, T. Engel, N. Schalch, A. Signer and Y. Ulrich, Bhabha scattering at NNLO with next-to-soft stabilisation, Phys. Lett. B 820 (2021) 136547 [arXiv:2106.07469] [INSPIRE].

[2] S. Actis, M. Czakon, J. Gluza and T. Riemann, Planar two-loop master integrals for massive Bhabha scattering: $N_{f}=1$ and $N_{f}=2$, Nucl. Phys. B Proc. Suppl. 160 (2006) 91 [hep-ph/0609051] [INSPIRE].

[3] S. Actis, M. Czakon, J. Gluza and T. Riemann, Two-loop fermionic corrections to massive Bhabha scattering, Nucl. Phys. B 786 (2007) 26 [arXiv:0704.2400] [INSPIRE].

[4] S. Actis, M. Czakon, J. Gluza and T. Riemann, Fermionic NNLO contributions to Bhabha scattering, Acta Phys. Polon. B 38 (2007) 3517 [arXiv:0710.5111] [InSPIRE].

[5] S. Actis, M. Czakon, J. Gluza and T. Riemann, Virtual hadronic and leptonic contributions to Bhabha scattering, Phys. Rev. Lett. 100 (2008) 131602 [arXiv:0711.3847] [InSPIRE].

[6] S. Actis, M. Czakon, J. Gluza and T. Riemann, Virtual Hadronic and Heavy-Fermion $\mathcal{O}\left(\alpha^{2}\right)$ Corrections to Bhabha Scattering, Phys. Rev. D 78 (2008) 085019 [arXiv:0807.4691] [INSPIRE].

[7] R. Bonciani, A. Ferroglia, P. Mastrolia, E. Remiddi and J.J. van der Bij, Planar box diagram for the $\left(N_{F}=1\right)$ two loop QED virtual corrections to Bhabha scattering, Nucl. Phys. B 681 (2004) 261 [Erratum ibid. 702 (2004) 364] [hep-ph/0310333] [INSPIRE].

[8] R. Bonciani, A. Ferroglia, P. Mastrolia, E. Remiddi and J.J. van der Bij, Two-loop $N_{F}=1$ QED Bhabha scattering differential cross section, Nucl. Phys. B 701 (2004) 121 [hep-ph/0405275] [INSPIRE].

[9] V.A. Smirnov, Analytical result for dimensionally regularized massive on-shell planar double box, Phys. Lett. B 524 (2002) 129 [hep-ph/0111160] [INSPIRE].

[10] G. Heinrich and V.A. Smirnov, Analytical evaluation of dimensionally regularized massive on-shell double boxes, Phys. Lett. B 598 (2004) 55 [hep-ph/0406053] [InSPIRE].

[11] M. Czakon, J. Gluza and T. Riemann, Master integrals for massive two-loop bhabha scattering in QED, Phys. Rev. D 71 (2005) 073009 [hep-ph/0412164] [INSPIRE].

[12] M. Czakon, J. Gluza and T. Riemann, Harmonic polylogarithms for massive Bhabha scattering, Nucl. Instrum. Meth. A 559 (2006) 265 [hep-ph/0508212] [INSPIRE].

[13] M. Czakon, J. Gluza and T. Riemann, On the massive two-loop corrections to Bhabha scattering, Acta Phys. Polon. B 36 (2005) 3319 [hep-ph/0511187] [InSPIRE].

[14] M. Czakon, J. Gluza and T. Riemann, The Planar four-point master integrals for massive two-loop Bhabha scattering, Nucl. Phys. B 751 (2006) 1 [hep-ph/0604101] [INSPIRE].

[15] M. Czakon, J. Gluza, K. Kajda and T. Riemann, Differential equations and massive two-loop Bhabha scattering: The B5l2m3 case, Nucl. Phys. B Proc. Suppl. 157 (2006) 16 [hep-ph/0602102] [INSPIRE].

[16] J.M. Henn and V.A. Smirnov, Analytic results for two-loop master integrals for Bhabha scattering I, JHEP 11 (2013) 041 [arXiv:1307.4083] [INSPIRE].

[17] J.A. Lappo-Danilevsky, Théorie algorithmique des corps de Riemann, Rec. Math. Moscou 34 (1927) 113.

[18] A.B. Goncharov, Multiple polylogarithms, cyclotomy and modular complexes, Math. Res. Lett. 5 (1998) 497 [arXiv:1105.2076] [INSPIRE].

[19] A.B. Goncharov, Multiple polylogarithms and mixed Tate motives, math/0103059 [INSPIRE]. 
[20] A.V. Kotikov, Differential equations method: New technique for massive Feynman diagrams calculation, Phys. Lett. B 254 (1991) 158 [INSPIRE].

[21] E. Remiddi, Differential equations for Feynman graph amplitudes, Nuovo Cim. A 110 (1997) 1435 [hep-th/9711188] [INSPIRE].

[22] T. Gehrmann and E. Remiddi, Differential equations for two loop four point functions, Nucl. Phys. B 580 (2000) 485 [hep-ph/9912329] [INSPIRE].

[23] J.M. Henn, Multiloop integrals in dimensional regularization made simple, Phys. Rev. Lett. 110 (2013) 251601 [arXiv: 1304.1806] [INSPIRE].

[24] M. Heller, A. von Manteuffel and R.M. Schabinger, Multiple polylogarithms with algebraic arguments and the two-loop EW-QCD Drell-Yan master integrals, Phys. Rev. D 102 (2020) 016025 [arXiv: 1907.00491] [INSPIRE].

[25] J. Broedel, C. Duhr, F. Dulat and L. Tancredi, Elliptic polylogarithms and iterated integrals on elliptic curves. Part I: general formalism, JHEP 05 (2018) 093 [arXiv: 1712.07089] [INSPIRE].

[26] J. Broedel, C. Duhr, F. Dulat and L. Tancredi, Elliptic polylogarithms and iterated integrals on elliptic curves II: an application to the sunrise integral, Phys. Rev. D 97 (2018) 116009 [arXiv: 1712.07095] [INSPIRE].

[27] J. Broedel, C. Duhr, F. Dulat, B. Penante and L. Tancredi, Elliptic symbol calculus: from elliptic polylogarithms to iterated integrals of Eisenstein series, JHEP 08 (2018) 014 [arXiv: 1803.10256] [INSPIRE].

[28] A.V. Smirnov and F.S. Chuharev, FIRE6: Feynman Integral REduction with Modular Arithmetic, Comput. Phys. Commun. 247 (2020) 106877 [arXiv: 1901.07808] [INSPIRE].

[29] P. Maierhöfer, J. Usovitsch and P. Uwer, Kira - A Feynman integral reduction program, Comput. Phys. Commun. 230 (2018) 99 [arXiv:1705.05610] [InSPIRE].

[30] J. Klappert, F. Lange, P. Maierhöfer and J. Usovitsch, Integral reduction with Kira 2.0 and finite field methods, Comput. Phys. Commun. 266 (2021) 108024 [arXiv:2008.06494] [INSPIRE].

[31] K.G. Chetyrkin and F.V. Tkachov, Integration by Parts: The Algorithm to Calculate $\beta$-functions in 4 Loops, Nucl. Phys. B 192 (1981) 159 [INSPIRE].

[32] F.V. Tkachov, A Theorem on Analytical Calculability of Four Loop Renormalization Group Functions, Phys. Lett. B 100 (1981) 65 [InSPIRE].

[33] N. Arkani-Hamed, J.L. Bourjaily, F. Cachazo and J. Trnka, Local Integrals for Planar Scattering Amplitudes, JHEP 06 (2012) 125 [arXiv:1012.6032] [INSPIRE].

[34] J. Henn, B. Mistlberger, V.A. Smirnov and P. Wasser, Constructing d-log integrands and computing master integrals for three-loop four-particle scattering, JHEP 04 (2020) 167 [arXiv: 2002.09492] [INSPIRE].

[35] T. Gehrmann, A. von Manteuffel, L. Tancredi and E. Weihs, The two-loop master integrals for $q \bar{q} \rightarrow V V$, JHEP 06 (2014) 032 [arXiv: 1404.4853] [INSPIRE].

[36] K.-T. Chen, Iterated path integrals, Bull. Am. Math. Soc. 83 (1977) 831 [InSPIRE].

[37] E. Remiddi and J.A.M. Vermaseren, Harmonic polylogarithms, Int. J. Mod. Phys. A 15 (2000) 725 [hep-ph/9905237] [INSPIRE].

[38] F. Brown and C. Duhr, A double integral of dlog forms which is not polylogarithmic, arXiv: 2006.09413 [INSPIRE]. 
[39] D. Festi and D. van Straten, Bhabha Scattering and a special pencil of K3 surfaces, Commun. Num. Theor. Phys. 13 (2019) 463 [arXiv:1809.04970] [InSPIRE].

[40] M. Besier, D. Van Straten and S. Weinzierl, Rationalizing roots: an algorithmic approach, Commun. Num. Theor. Phys. 13 (2019) 253 [arXiv:1809.10983] [InSPIRE].

[41] M. Besier, P. Wasser and S. Weinzierl, RationalizeRoots: Software Package for the Rationalization of Square Roots, Comput. Phys. Commun. 253 (2020) 107197 [arXiv: 1910.13251] [INSPIRE].

[42] M.R. Besier, Rationalization Questions in Particle Physics, Ph.D. Thesis, Johannes Gutenberg-Universität Mainz (2020) [DOI] [INSPIRE].

[43] M. Besier and D. Festi, Rationalizability of square roots, arXiv:2006.07121 [INSPIRE].

[44] D. Festi and A. Hochenegger, Rationalizability of field extensions, arXiv:2106.05621 [INSPIRE].

[45] M. Heller, Planar two-loop integrals for $\mu \mathbf{e}$ scattering in QED with finite lepton masses, arXiv:2105.08046 [INSPIRE].

[46] P.A. Kreer and S. Weinzierl, The H-graph with equal masses in terms of multiple polylogarithms, Phys. Lett. B 819 (2021) 136405 [arXiv:2104.07488] [INSPIRE].

[47] F. Brown and A. Levin, Multiple Elliptic Polylogarithms, arXiv:1110.6917.

[48] J. Broedel, C.R. Mafra, N. Matthes and O. Schlotterer, Elliptic multiple zeta values and one-loop superstring amplitudes, JHEP 07 (2015) 112 [arXiv:1412.5535] [INSPIRE].

[49] J. Broedel, C. Duhr, F. Dulat, B. Penante and L. Tancredi, Elliptic Feynman integrals and pure functions, JHEP 01 (2019) 023 [arXiv: 1809.10698] [INSPIRE].

[50] S. Pozzorini and E. Remiddi, Precise numerical evaluation of the two loop sunrise graph master integrals in the equal mass case, Comput. Phys. Commun. 175 (2006) 381 [hep-ph/0505041] [INSPIRE].

[51] U. Aglietti, R. Bonciani, L. Grassi and E. Remiddi, The Two loop crossed ladder vertex diagram with two massive exchanges, Nucl. Phys. B $\mathbf{7 8 9}$ (2008) 45 [arXiv:0705.2616] [INSPIRE].

[52] M. Caffo, H. Czyz, M. Gunia and E. Remiddi, BOKASUN: A Fast and precise numerical program to calculate the Master Integrals of the two-loop sunrise diagrams, Comput. Phys. Commun. 180 (2009) 427 [arXiv:0807.1959] [INSPIRE].

[53] R. Bonciani, G. Degrassi, P.P. Giardino and R. Gröber, A Numerical Routine for the Crossed Vertex Diagram with a Massive-Particle Loop, Comput. Phys. Commun. 241 (2019) 122 [arXiv: 1812.02698] [INSPIRE].

[54] F. Moriello, Generalised power series expansions for the elliptic planar families of Higgs + jet production at two loops, JHEP 01 (2020) 150 [arXiv:1907.13234] [INSPIRE].

[55] R. Bonciani et al., Evaluating a family of two-loop non-planar master integrals for Higgs + jet production with full heavy-quark mass dependence, JHEP 01 (2020) 132 [arXiv: 1907.13156] [INSPIRE].

[56] S. Abreu, H. Ita, F. Moriello, B. Page, W. Tschernow and M. Zeng, Two-Loop Integrals for Planar Five-Point One-Mass Processes, JHEP 11 (2020) 117 [arXiv:2005.04195] [INSPIRE].

[57] M. Hidding, DiffExp, a Mathematica package for computing Feynman integrals in terms of one-dimensional series expansions, Comput. Phys. Commun. 269 (2021) 108125 [arXiv: 2006.05510] [INSPIRE]. 
[58] A. Pak and A. Smirnov, Geometric approach to asymptotic expansion of Feynman integrals, Eur. Phys. J. C 71 (2011) 1626 [arXiv: 1011.4863] [INSPIRE].

[59] B. Jantzen, A.V. Smirnov and V.A. Smirnov, Expansion by regions: revealing potential and Glauber regions automatically, Eur. Phys. J. C 72 (2012) 2139 [arXiv:1206.0546] [INSPIRE].

[60] A.V. Smirnov, FIESTA4: Optimized Feynman integral calculations with GPU support, Comput. Phys. Commun. 204 (2016) 189 [arXiv:1511.03614] [INSPIRE].

[61] A.V. Smirnov, FIESTA, a new release, to appear.

[62] C.W. Bauer, A. Frink and R. Kreckel, Introduction to the GiNaC framework for symbolic computation within the $C++$ programming language, J. Symb. Comput. 33 (2002) 1 [cs/0004015] [INSPIRE].

[63] J. Vollinga and S. Weinzierl, Numerical evaluation of multiple polylogarithms, Comput. Phys. Commun. 167 (2005) 177 [hep-ph/0410259] [INSPIRE].

[64] F.C.S. Brown, Multiple zeta values and periods of moduli spaces $\mathfrak{M}_{0, n}$, Annales Sci. Ecole Norm. Sup. 42 (2009) 371 [math/0606419] [INSPIRE].

[65] F. Brown, The Massless higher-loop two-point function, Commun. Math. Phys. 287 (2009) 925 [arXiv: 0804.1660] [INSPIRE].

[66] C. Anastasiou, C. Duhr, F. Dulat and B. Mistlberger, Soft triple-real radiation for Higgs production at N3LO, JHEP 07 (2013) 003 [arXiv:1302.4379] [INSPIRE].

[67] J. Ablinger, J. Blümlein, C. Raab, C. Schneider and F. Wißbrock, Calculating Massive 3-loop Graphs for Operator Matrix Elements by the Method of Hyperlogarithms, Nucl. Phys. B $\mathbf{8 8 5}$ (2014) 409 [arXiv: 1403.1137] [INSPIRE].

[68] C. Bogner and F. Brown, Feynman integrals and iterated integrals on moduli spaces of curves of genus zero, Commun. Num. Theor. Phys. 09 (2015) 189 [arXiv:1408.1862] [INSPIRE].

[69] E. Panzer, Algorithms for the symbolic integration of hyperlogarithms with applications to Feynman integrals, Comput. Phys. Commun. 188 (2015) 148 [arXiv:1403.3385] [InSPIRE].

[70] C. Duhr and F. Dulat, PolyLogTools - polylogs for the masses, JHEP 08 (2019) 135 [arXiv: 1904.07279] [INSPIRE].

[71] M. Walden and S. Weinzierl, Numerical evaluation of iterated integrals related to elliptic Feynman integrals, Comput. Phys. Commun. 265 (2021) 108020 [arXiv:2010.05271] [INSPIRE].

[72] A.B. Goncharov, Galois symmetries of fundamental groupoids and noncommutative geometry, Duke Math. J. 128 (2005) 209 [math/0208144] [INSPIRE].

[73] F. Brown, On the decomposition of motivic multiple zeta values, arXiv:1102.1310 [INSPIRE].

[74] C. Duhr, Hopf algebras, coproducts and symbols: an application to Higgs boson amplitudes, JHEP 08 (2012) 043 [arXiv: 1203.0454] [INSPIRE].

[75] A.B. Goncharov, Geometry of configurations, polylogarithms, and motivic cohomology, Adv. Math. 114 (1995) 197.

[76] A.B. Goncharov, M. Spradlin, C. Vergu and A. Volovich, Classical Polylogarithms for Amplitudes and Wilson Loops, Phys. Rev. Lett. 105 (2010) 151605 [arXiv:1006.5703] [INSPIRE].

[77] C. Duhr, H. Gangl and J.R. Rhodes, From polygons and symbols to polylogarithmic functions, JHEP 10 (2012) 075 [arXiv:1110.0458] [INSPIRE]. 